\title{
Estimation of Directional Wave Spectrum Using Measurement Array Pressure Data on Bottom-Mounted Offshore Structure in Incident and Diffracted Wave Field
}

\author{
Xiaodong Song, ${ }^{1,2}$ Zilong Ti $\mathbb{D}^{1}{ }^{1}$ and Yuanzhou Zhou ${ }^{1}$ \\ ${ }^{1}$ Department of Bridge Engineering, Southwest Jiaotong University, Chengdu 610031, China \\ ${ }^{2}$ China Railway Eryuan Engineering Group Co. Ltd, Chengdu, China \\ Correspondence should be addressed to Zilong Ti; swjtutzl@126.com
}

Received 24 November 2021; Revised 16 December 2021; Accepted 5 January 2022; Published 27 February 2022

Academic Editor: Jialong Jiao

Copyright (C) 2022 Xiaodong Song et al. This is an open access article distributed under the Creative Commons Attribution License, which permits unrestricted use, distribution, and reproduction in any medium, provided the original work is properly cited.

Determination of the directional wave spectrum which offshore structures actually encounter is essential for multiple applications including wave-induced load and vibration evaluation, and hence becomes a fundamental task in ocean engineering. Due to the wave diffraction effect, wave field around an offshore structure is the mixture of incident wave components and diffracted wave components. Estimating directional wave spectrum in diffracted wave field significantly differs from the occasion in undisturbed waves since the amplitude and phase relationship between the incident and diffracted waves are coupled, and therefore making the conventional approach not applicable. In this study, the diffraction wave theory is introduced into the estimation of directional wave spectrum to consider the effect of diffracted waves using array pressure data from existing pressure gauges on structures. Considering the performance of the presented approach under scenarios with various gauge arrays, different directions, and spreading coefficients, multiple levels of background noise are evaluated and discussed, respectively. The presented approach is also deployed into an in-situ measurement application on a marine structure and compared with wave observation data to test its feasibility in engineering practice. In general, the presented approach can reasonably estimate the directional wave spectrum and show advantages over the conventional approach in which the diffraction effect is excluded.

\section{Introduction}

The three-dimensional wave spectrum, i.e., directional wave spectrum, providing the basic information including energy and direction distributions for stochastic waves, is one of the most fundamental properties in offshore engineering. Measurement and determination of the directional wave spectrum for the offshore structures is an engineering premise of multiple applications, including the evaluation of hydrodynamic loads [1], wave-induced vibration [2], etc., and hence becomes a primary task in the health monitoring and maintenance of offshore structures. Conventionally, estimation of directional wave spectrum is usually performed using measurement data collected by spatial array or pitch-and-roll buoys. However, after the structure is built in place, the local wave field is disturbed by the presence of structure due to the wave diffraction effect [3]. Wave diffraction refers to a particular phenomenon when propagating waves encounter obstacles, and the obstacle surface may, depending on the obstacle size, induce diffracted waves. Conventional methods using measurement data such as array wave elevations [4] or array wave pressures [5] to estimate the directional wave spectrum are based on the assumption that the wave field is an undisturbed condition, and therefore all wave components are independent and random. However, the disturbed wave field, containing the incident and diffracted wave components, brings challenges to the estimation of directional wave spectrum because the relationship between diffracted and incident waves is synchronized and coupled. Some previous studies [6-8] that investigated the disturbed wave field due to wave reflection effect in the laboratory shows that the disturbed wave components may impose significant deviations in the spectral density and direction results and hence the 
conventional methods for undisturbed wave field are not applicable. Accurate estimation of the actually encountering directional wave spectrum for offshore structures in the incident and diffracted wave field could benefit the scientific and engineering practice including real-time load evaluation [9], structural monitoring [10], motion control, etc., and therefore, is essential to study. However, limited literature focuses on this issue and still lacks in-depth investigation.

Wave spectra are usually present in either two-dimension or three-dimension forms. A two-dimension wave spectrum only contains energy distribution in the frequency domain and is easily estimated by using measurement data from single-point gauges. Stochastic waves have an inherent nature of multidirections. A three-dimension wave spectrum could provide energy and directional distributions while it is usually more difficult to obtain. In order to capture the directional information in stochastic waves, researchers spend efforts on improving both measurement techniques $[11,12]$ and estimation methods [5, 13]. In general, two mainstream approaches exist to obtain the directional wave spectrum. One way is to deploy single-point devices such as multifunctional buoys, which can collect velocity, pressure, motion data, and use various algorithms to analyze the cross-spectral relationship between them to approximate the directional wave spectrum [14]. This approach is direct and convenient, but the withdraws are also obvious that the costs on the devices and installation are usually considerable. Furthermore, these single-point devices still lack the ability to separate the incident wave components from the disturbed wave field due to insufficient information on multiple locations. Another way is to use multiple array gauges, e.g., array wave gauges and array pressure gauges, to synchronously record data and estimate the directional wave pressure by analyzing the cross-spectral information between them $[5,6]$. While less convenient compared with the first approach, this way is easier to implement and widely used by researchers. Studies $[6,15]$ show that by utilizing appropriate modifications, this technique also has the ability to separate reflected wave components in disturbed wave fields and gives appreciable estimated results. Another advantage of using array gauge measurement is that in present time, the array gauges are usually a part of structural health monitoring (SHM) system, such as pressure gauges embedded on structures to observe the hydrodynamic load and hence there is no need to spend additional efforts and expense on devices and their installation. In light of this statement, this study focuses on utilizing array pressure data from existing pressure gauges embedded on structures to estimate the directional wave spectra.

Various methods are proposed to estimate the directional spectrum from array measurement data. A pioneering attempt was presented in the literature [13] by using Direct Fourier Transform method to analyze the spectral information and give a direct approximation of the incident wave spectrum. Howell and Gary [5] invented a measurement equipment by embedding six pressure gauges on a hexagonal frame to simultaneously measure wave pressure and make estimations from array pressure data. To make the estimation more robust, MLM (Maximum Likelihood Method)
[16] and parametric method [17] were proposed and aimed to improve the overall accuracy in the final guess of the spectra from cross-spectral results. Literature [18] investigated the directional spectra using a eight-wave-probe array and presented the computational routine and preliminary validation. To sum up, the studies above only consider the estimation in the undisturbed wave field. Literature $[6,7]$ pointed out that influenced by reflected or refracted waves, the wave field may contain different wave components and the standard method cannot separate these phase-related components and therefore causes errors in spectrum estimation. Isobe [15] proposed a modified MLM method to consider the reflected waves from vertical walls by adding a phase-related term in the governing equations for spectrum estimation. Later, based on this study, some researchers $[7,19]$ further isolated the reflected components and used the approach to determinate the reflection coefficients. Study [20] proposed a new method to isolate incident and reflected spectra using a gauge array in a multidirectional wave basin. This series of studies indicate that separating the wave components in disturbed wave field is essential in spectrum estimation and provide a basic methodology for a relative problem. Another practical mainstream for spectra estimation is to build the relationship between the incident wave and global structural responses [21-23]. However, regarding estimating the direction wave spectrum of wave field with the incident and diffracted waves around offshore structures, until now, there is very limited literature addressing this issue and lacks useful solutions.

In the present study, the diffraction wave theory is introduced into the estimation of directional wave spectrum to consider the effect of diffracted waves using array pressure data from existing pressure gauges on structures. Two numerical experiments are conducted as proof-of-concept studies to test and evaluate the feasibility of the presented approach under various structures and wave scenarios. The performance of presented approach using various gauge arrays, different wave directions and spreading coefficients and multiple levels of background noise are evaluated and discussed, respectively. The presented approach is also deployed into an in-situ measurement application and compared with wave observation data to test its validity in engineering practice. In the end, some main conclusions associated with limitations and further works regarding the presented approach are drawn.

\section{Methodology}

2.1. Fundamentals. Stochastic waves are usually presented as a sum of infinite cosine waves and the stochastic wave surface elevation $\eta$ at location coordinate $\boldsymbol{x}$ can be written in the form of spectral presentation:

$$
\eta(x, t)=\int_{\sigma} \int_{\vec{k}} e^{i(\vec{k} x-\omega t)} Z(d \vec{k}, d \omega)
$$

where $k$ is the wave number vector and $\omega$ is the wave frequency. $Z(d \vec{k}, d \sigma)$ denotes the wave energy within the wave 
number range of $[\vec{k}, \vec{k}+d \vec{k}]$ and wave frequency range of $[\omega, \omega+d \omega]$ and is a complex number of which absolute value is the wave amplitude and argument is the wave phase. The cross-energy wave spectrum $\Phi_{m n}$ at location coordinate $\vec{x}_{m} \vec{x}_{n}$ can be expressed as the Fourier transform of the product between wave components as follows:

$$
\Phi_{m n}=\int_{0}^{2 \pi} e^{-i \vec{k}}\left(\vec{x}_{m^{-}} \vec{x}_{n}\right)_{E}(\omega, \theta) d \theta
$$

where $E(\omega, \theta)$ denotes the directional wave spectrum. In order to separate the direction and energy information, the directional wave spectrum $E(\omega, \theta)$ is usually expressed as the product of spreading function $D(\omega, \theta)$ and spectral density $S(\omega)$ as follows:

$$
\Phi_{m n}=S(\omega) \int_{0}^{2 \pi} e^{-i \vec{k}\left(\vec{x}_{m}-\vec{x}_{n}\right)} D(\omega, \theta) d \theta
$$

The equation above describes the basic mathematical relationship between wave cross-spectrum, wave spectral density, and spreading function, which is the foundational formulation for spectrum estimation. Furthermore, if the given data is wave pressure instead of wave surface elevation, by inserting the relationship between wave pressure and surface elevation of Airy wave theory, the cross-energy wave pressure spectrum $\Phi_{m n, p}$ becomes as follows:

$$
\Phi_{m n, p}=S(\omega) \int_{0}^{2 \pi}\left\{\rho g \frac{\operatorname{ch}[\vec{k}(d+z)]}{\operatorname{ch}(\vec{k} d)}\right\}^{2} e^{-i \vec{k}\left(\vec{x}_{m^{-}}-\vec{x}_{n}\right)} D(\omega, \theta) d \theta
$$

Howell [5] uses (4) as the basic formulation to estimate the undisturbed incident wave field using array pressure gauges fixed on a hexagonal frame. The theories above have a tacit assumption that all the wave components are independent and random. However, when the wave field consists of both incident and diffracted waves, the relationship between the incident and diffracted components is determined and therefore, (4) is invalid and needs modification.
The incident wave pressure $p_{i}(x, t)$ of the stochastic wave series described in (1) reads as follows:

$$
p_{i}(x, t)=\int_{\omega} \int_{\vec{k}} \rho g \frac{\operatorname{ch}[\vec{k}(d+z)]}{\operatorname{ch}(\vec{k} d)} e^{i(\vec{k} x-\omega t)} Z(d \vec{k}, d \omega) .
$$

Wave diffraction theory of the first order is introduced to mathematically describe the diffraction effect. According to wave diffraction theory [3], as demonstrated in Figure 1, the actual wave field around the structure (Figure 1(c)) is the superposition of the incident wave field (Figure 1(a)) and the diffracted wave field (Figure 1(b)). Therefore, the actual disturbed wave pressure on the structure is the combination of incident wave pressure and diffracted wave pressure which reads as follows:

$$
\begin{aligned}
p_{i}(x, t)+p_{d}(x, t)= & \int_{\omega} \int_{\vec{k}} \rho g \frac{\operatorname{ch}[\vec{k}(d+z)]}{\operatorname{ch}(\vec{k} d)} e^{i(\vec{k} x-\omega t)} Z(d \vec{k}, d \omega) \\
& +\int_{\sigma} \int_{\vec{k}_{d}} \rho g \frac{\operatorname{ch}\left[\overrightarrow{k_{d}}(d+z)\right]}{\operatorname{ch}\left(\overrightarrow{k_{d}} d\right)} e^{i\left(\overrightarrow{k_{d}} x-\omega t\right)} Z\left(d \overrightarrow{k_{d}}, d \omega\right),
\end{aligned}
$$

where the subscript $i$ and $d$ denote the incident and diffracted components. In principle, any signals related to waves, i.e., elevation, pressure, response, etc., may be utilized as the product of the incident component and a complexvalued transfer function. Therefore, by substituting (6) into (4), the cross-energy spectrum of wave pressure on the structure becomes as follows:

$$
\Phi_{m n, p}=E(\omega) \int_{0}^{2 \pi} H_{m} H_{n}^{*} e^{-i \vec{k}\left(\vec{x}_{m}-\vec{x}_{n}\right)} D(\omega, \theta) d \theta
$$

$H$ is the complex-valued transfer function which reads as follows:

$$
H(\vec{k}, \omega)=1+\frac{\operatorname{ch}\left[\overrightarrow{k_{d}}(d+z)\right]}{\operatorname{ch}\left(\overrightarrow{k_{d}} d\right)} e^{i\left(\overrightarrow{k_{d}} x-\omega t\right)} \frac{Z\left(d \overrightarrow{k_{d}}, d \omega\right)}{\left\{\operatorname{ch}[\vec{k}(d+z)] / \operatorname{ch}(\vec{k} d) e^{i(\vec{k} x-\omega t)} Z(d \vec{k}, d \omega)\right\}} .
$$

A prior task in the calculation of (7) is the determination of the transfer function $H$. This transfer function, of which physical definition is the mapping from incident wave elevation to wave pressure on the structure, can be directly investigated by either analytical solutions for structures with regular geometry such as circular cylinders [3], or numerical approaches such as the boundary element method (BEM) [24]. The details in the determination of transfer function for specific structures will be described in the following sections.
By using (7) as the governing formulation and appropriate numerical method, the directional spectrum can be approximated by using a finite number of pressure data on the offshore structure.

2.2. Numerical Solution. Theoretically speaking, the calculation of directional spectrum using (7) requires pressure data at infinite locations around the structures, which is very 


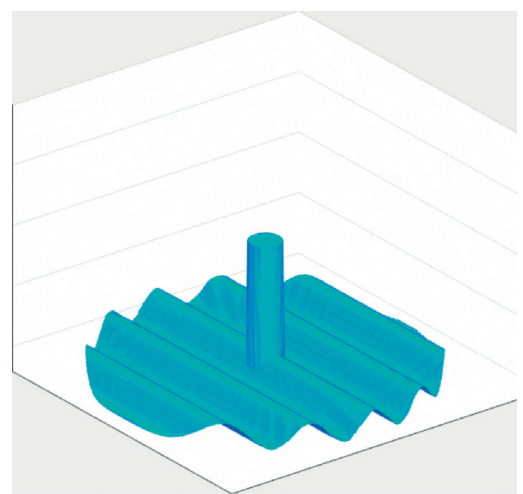

(a)

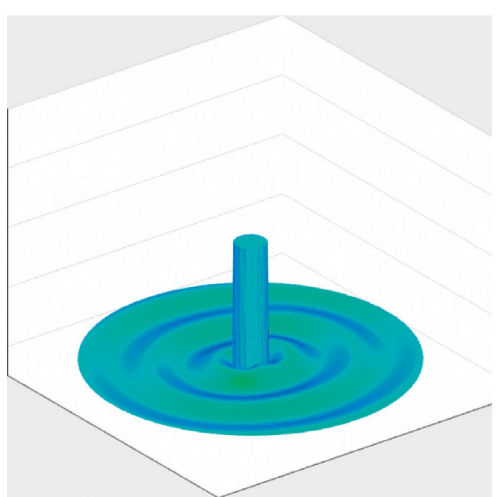

(b)

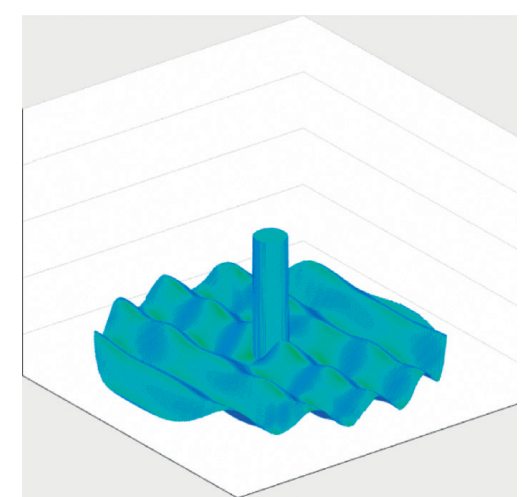

(c)

Figure 1: Wave field around a cylinder: (a) incident wave field; (b) diffracted wave field due to the presence of cylinder; (c) actual wave field around a cylinder as the superposition of (a) and (b).

difficult in practice. Researchers developed various numerical solutions to approximate the accurate spectra using data from an only a finite number of locations, such as Maximum Likelihood Method (MLM), Maximum Entropy Method (MEM) [25], and Bayesian approach (BDM) [4]. These methods may deviate in accuracy and have their specific requirements on array pattern, gauge quantity, etc. According to previous studies [4, 20], by employing a
Bayesian approach to approximate the sample data by a most reasonable model, BDM can give the direction spectrum results with appreciable accuracy and has an inherent good ability to resist signal noise. Therefore, the Bayesian approach BDM is employed in this study. A brief description of using the Bayesian procedure to approximate the direction spectrum is introduced. In BDM, the basic formulation of the cross-energy spectrum is rewritten as follows:

$$
\begin{aligned}
\varphi_{i} & =\int_{0}^{2 \pi} H_{i}(\omega, \theta) G(\theta \mid \omega) d \theta, \quad(i=1,2, \ldots, N) \\
H_{i}(\omega, \theta) & =H_{m}(\omega, \theta) H_{n} *(\omega, \theta) \frac{\left[\cos \left(k x_{m n} \cos \theta+k y_{m n} \sin \theta\right)-i \sin \left(k x_{m n} \cos \theta+k y_{m n} \sin \theta\right)\right]}{\sqrt{\varphi_{m m}(f) \varphi_{m n}(f)}} \\
G(\theta \mid \omega) & =\frac{S(\omega, \theta)}{S(\omega)} s
\end{aligned}
$$

where $N=M(M+1) / 2$ and $M$ is the number of wave signals. $\theta$ is the wave direction. Assuming the directional spreading function $G(\theta \mid \omega)$ is divided into $K$ partitions and is approximated as follows:

$$
\begin{aligned}
G\left(\theta_{k} \mid \omega\right) & =\sum_{k=1}^{K} \exp \left[x_{k}(\omega)\right] I_{k}(\theta), \quad(k=1,2, \ldots, K), \\
I_{k}(\theta) & = \begin{cases}1, & (k-1) \Delta \theta \leq \theta \leq k \Delta \theta \\
0 & \end{cases}
\end{aligned}
$$

Substituting (12) into (9), after some mathematical manipulations and inserting an error term $\varepsilon_{i}$, the crossenergy spectrum becomes as follows:

$$
\varphi_{i}=\sum_{k=1}^{K} \alpha_{i k} \exp \left(x_{k}\right)+\varepsilon_{i}, \quad(i=1,2, \ldots, 2 N) .
$$

The subscripts $i=1$ to $\mathrm{N}$ and $N+1$ to $2 \mathrm{~N}$ represent real parts and imaginary parts of the complex cross-energy spectrum $\varphi_{i}$ and $\alpha_{i k}$, respectively. The error term $\varepsilon_{i}$ is assumed to have the probability of their occurrence expressed by the normal distribution having the mean value of 0 and the variance $\sigma^{2}$. The likelihood function of $x_{k}$ and $\sigma^{2}$ is given by the following:

$$
L\left(x_{1}, x_{2}, \ldots, x_{k} ; \sigma^{2}\right)=\frac{1}{\left(2 \pi \sigma^{2}\right)^{N}} \exp \left\{-\frac{1}{2 \sigma^{2}} \sum_{i=1}^{2 N}\left[\varphi_{i}-\sum_{k=1}^{K} \alpha_{i k} \exp \left(x_{k}\right)^{2}\right]\right\}
$$


With a basic assumption that the wave component is independent of each other in the frequency domain and the directional function is a continuous and smooth one, it yields a limiting condition of $x_{k}$ which reads as follows:

$$
x_{k}-2 x_{k-1}+x_{k-2} \approx 0
$$

To make the directional function smoother, (13) is equivalent to minimizing the following equation:

$$
\sum_{k=1}^{K}\left(x_{k}-2 x_{k-1}+x_{k-2}\right)^{2}
$$

Therefore, the estimation of directional spreading function should maximize the likelihood of (12) and minimize that of (14) at the same time, which is equivalent to maximizing the following equation:

$\sum_{i=1}^{2 N}\left[\varphi_{i}-\sum_{k=1}^{K} \alpha_{i k} \exp \left(x_{k}\right)^{2}\right]+u^{2}\left[\sum_{k=1}^{K}\left(x_{k}-2 x_{k-1}+x_{k-2}\right)^{2}\right]$,

where $u^{2}$ is an additional hyperparameter. The introduction of this hyperparameter aims to meet the requirement of maximizing the likelihood (12) and minimizing that of (14) simultaneously. The optimal solution of $u^{2}$ and $\sigma^{2}$ should minimize Akaike's Bayesian Information Criterion (ABIC) to achieve the most suitable balance between smoothness and continuousness. Consequently, the resultant directional function is the potentially most suitable one. The ABIC reads as follows:

$$
\mathrm{ABIC}=-2 \ln \int L\left(x, \sigma^{2}\right) p\left(x \mid u^{2}, \sigma^{2}\right) d x,
$$

where $p\left(x \mid u^{2}, \sigma^{2}\right)$ is the prior distribution of $x=\left(x_{1}, x_{2}, \ldots, x_{k}\right)$ :

$p\left(x \mid u^{2}, \sigma^{2}\right)=\left(\frac{u}{\sqrt{2 \pi} \sigma}\right)^{K} \exp \left[-\frac{u^{2}}{2 \sigma^{2}} \sum_{k=1}^{K}\left(x_{k}-2 x_{k-1}+x_{k-2}\right)^{2}\right]$.
The hyperparameter $u$ and $\sigma^{2}$ can be numerically solved so that the approximation of directional spreading function and spectral density is determined. A flowchart demonstrating the procedure of the proposed approach is shown in Figure 2 .

\section{Numerical Validation and Application}

The most convenient and valid way to test the estimation method of wave spectrum is via numerical experiments and is widely adopted for validation purposes in spectrum estimation $[6,25,26]$. Therefore, two numerical experiments are conducted on structures with various geometries to test the performance of the proposed approach on directional spectrum estimation.

3.1. Numerical Experiment 1: Circular Cylinder. Circular cylinder is one of the most common offshore structures either in aspect of scientific research or engineering practice and is one of the very few geometries with analytical solutions for wave diffraction problems. First, the analytical solution of the diffraction problem for the circular cylinder is introduced. As demonstrated in Figure 3(a), regarding a bottom-fixed circular cylinder in regular wave field with a wave height of $H$, frequency $\omega$, the incident wave surface elevation reads as follows:

$$
\eta(x, t)=\frac{H_{w}}{2} e^{i k x-i \omega t} .
$$

The disturbed wave field, i.e., superposition of incident and diffracted waves, is written in the polar coordinate system as follows:

$$
\eta(r, \theta, t)=\frac{H_{w}}{2}\left\{\sum_{m=0}^{\infty} \beta_{m}\left[J_{m}(k r)-\frac{J_{m}{ }^{\prime}(k a)}{H_{m}{ }^{\prime}(k a)} H_{m}(k r)\right] \cos (m \theta) e^{-i \omega t}\right\} .
$$

Using relationship between wave pressure $p$ and surface elevation $\eta$ described by Airy wave theory, (19) becomes as follows:

$$
p(r, \theta, z, t)=\rho g \frac{H_{w}}{2} \frac{\cosh (k z)}{\cosh (k d)}\left\{\sum_{m=0}^{\infty} \beta_{m}\left[J_{m}(k r)-\frac{J_{m}{ }^{\prime}(k a)}{H_{m}{ }^{\prime}(k a)} H_{m}(k r)\right] \cos (m \theta) e^{-i \omega t}\right\}
$$




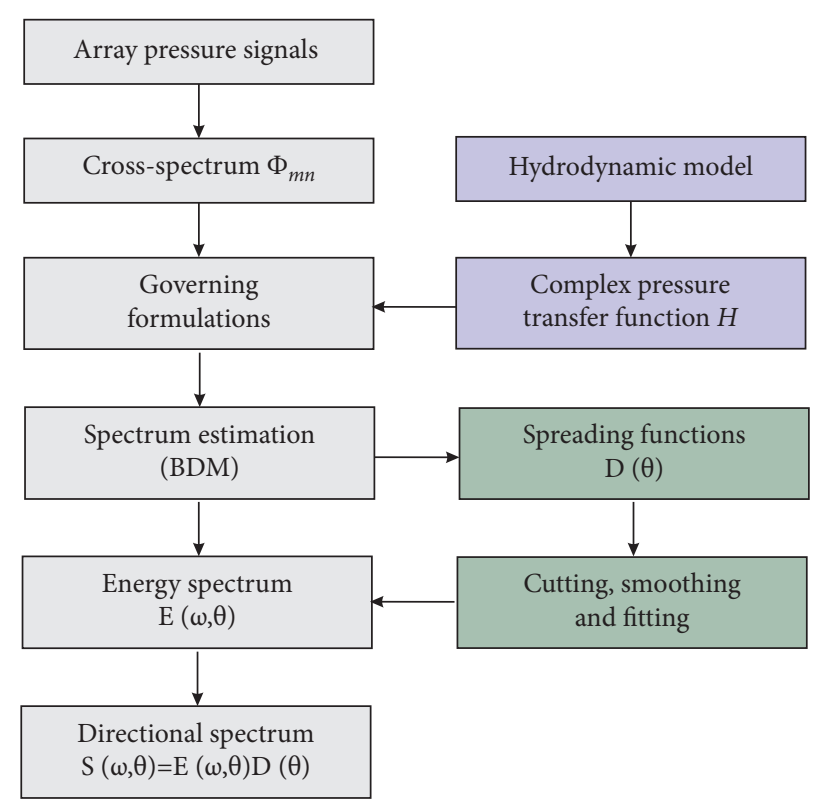

Figure 2: Flowchart of the proposed approach for wave spectrum estimation.

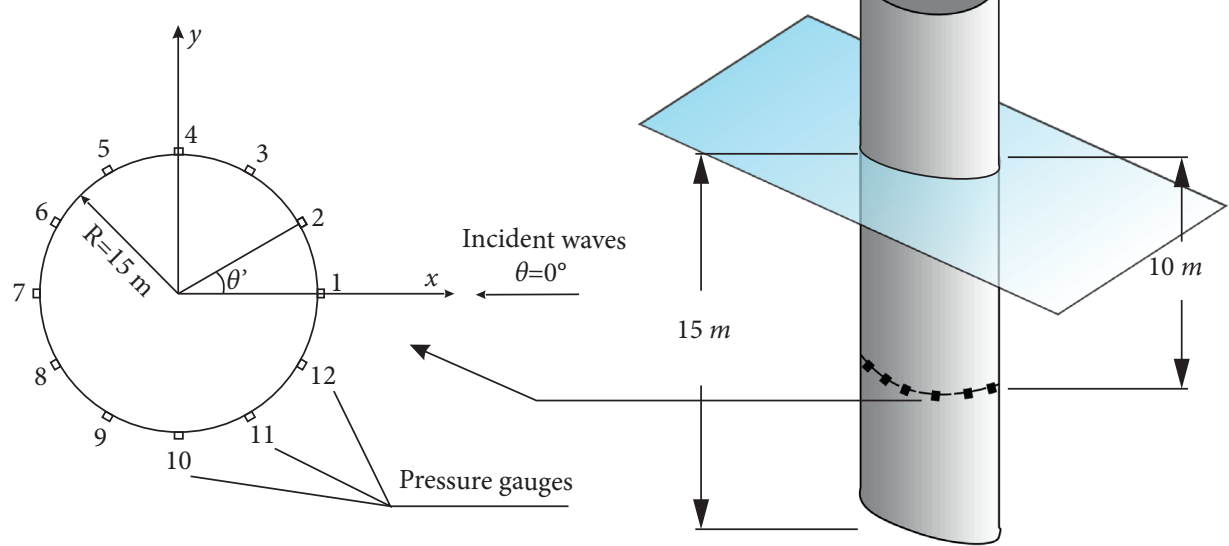

FIGURE 3: Structure layout and deployment of pressure gauges.

Assuming a pressure gauge is installed at location $(x, y, z)$ in the Cartesian coordinate system and is converted to the polar coordinate system as $\left(r, \theta^{\prime}, z\right)$. If the incident wave direction is $\theta$, the transfer function $H$ of wave pressure at the gauge reads as follows:

$$
H(\theta, k)=\rho g \frac{\cosh (k z)}{\cosh (k d)} \frac{\left\{\sum_{m=0}^{\infty} \beta_{m}\left[J_{m}(k a)-J_{m}{ }^{\prime}(k a) / H_{m}{ }^{\prime}(k a) H_{m}(k a)\right] \cos \left[m\left(\pi-\left(\theta-\theta^{\prime}\right)\right)\right]\right\}}{e^{i a \cos \left(\theta-\theta^{\prime}\right)}}
$$

Wave number $k$ can be directly determined by frequency $\omega$ using dispersion relation and hence transfer function is also the function of wave frequency and direction, i.e., $H(\theta, \omega)$. Once the transfer function $H$ is given, the directional wave spectrum can be estimated using (7) associated with the numerical procedure of BDM.
In the numerical experiment, the stochastic incident waves are determined by a JONSWAP spectrum associated with the cos 2 s-type directional spreading function. The spreading function writes as follows:

$$
D(\theta)=G_{0} \cos ^{2 s}\left(\frac{\theta-\theta_{0}}{2}\right)=\frac{\Gamma^{2}(s+1)}{\Gamma(2 s+1)} \cos ^{2 s}\left(\frac{\theta-\theta_{0}}{2}\right),
$$


where $s$ is the spreading parameter and $\theta_{0}$ is the main wave direction. The input spectral parameters in the numerical experiment are significant wave height of $H_{s}=3.5 \mathrm{~m}$, peak wave period $T_{p}=9 \mathrm{~s}$, peak enhancement factor $\gamma=3.3$, which represents an observed extreme wave condition in nearshore area [27]. Main wave direction $\theta_{0}=0^{\circ}$ and spreading parameter $s=15$. Twelve pressure gauges are evenly deployed at the depth of $-10 \mathrm{~m}$ around the cylinder, of which radius is $15 \mathrm{~m}$ and the water depth is $15 \mathrm{~m}$, as illustrated in Figure 3.

In the numerical experiment, stochastic wave pressures on the cylinder are generated using the following formulation:

$p_{i}(x, y, z, t)=\sum_{n=1}^{N} H_{i}\left(\omega_{n}, \theta_{n}, z\right) e^{i\left(k_{n}\left(x \cos \theta_{n}+y \sin \theta_{n}\right)-\omega_{n} t+\sigma_{n}\right)}$,

where $H$ is the analytical pressure transfer function (24) and $i$ denotes the $i^{\text {th }}$ wave pressure gauge and $n$ denotes the $n^{\text {th }}$ random components adopted. The sampling frequency is set at $3 \mathrm{~Hz}$ and the simulation duration is 600s. Afterward, the generated pressure data are fed to the presented approach to perform the spectrum estimation and the results are discussed.

\subsection{Numerical Experiment 2: Quasirectangle Cofferdam.} Cofferdam is a common facility to create a dry and safe construction environment when the foundation of offshore structures takes place below the water surface [27]. To perform accurate evaluation of hydrodynamic loads and structural safety, it is essential to estimate the wave spectra which the cofferdam is actually encountering. A quasirectangle cofferdam with rounded corners used for cross-sea bridge foundation construction is taken as an example in this study, as shown in Figure 4. The dimensions of the cofferdam are $23 \times 14.8 \times 12.3 \mathrm{~m}$. The cofferdam bottom is set at $-4.4 \mathrm{~m}$ and the water depth is $10 \mathrm{~m}$. Eleven pressure gauges, which are a part of the structural health monitoring system, are embedded on the surface to monitor the real-time hydrodynamic load of the cofferdam. Due to the large scale of the structure, diffracted waves significantly affect the wave field which brings challenges to the accurate estimation of the directional wave spectrum.

Because of the complex geometry, the diffracted waves are difficult to obtain analytically and the numerical method, i.e., boundary element method (BEM), is hence employed to calculate the transfer function of diffracted waves and pressures. In BEM, a particular Green function that satisfies the boundary conditions in wave diffraction problems is introduced and the three-dimensional integration problem is reduced to a two-dimensional integration problem. The body surface is discretized into finite quadrilateral elements and the potentials and their derivations are calculated to evaluate the wave pressure. The details of employed BEM can be found in authors' previous article [24] and for the sake of conciseness, the details are not listed here. The BEM model and panel mesh of the cofferdam is illustrated in
Figure 5(a). A total number of 11 complex pressure transfer functions are calculated using the BEM model and the real and imaginary part of the complex transfer function of wave pressure at \#4 gauge on the cofferdam are shown as an example in Figures 5(c) and 5(d).

The stochastic wave pressure signals are simulated using (23) in Section 3.1 with the complex transfer function calculated by the BEM model. The still water level is $+2.37 \mathrm{~m}$, which represents a measured extreme wave condition. The input spectral parameters remain the same as employed in Section 3.1 and different main wave directions of $30^{\circ}, 45^{\circ}$, and $60^{\circ}$ are applied and tested. The sampling frequency is $3 \mathrm{~Hz}$ and the simulation time duration is $600 \mathrm{~s}$. The generated pressure data are fed to the presented approach to perform the spectrum estimation and the results are discussed.

3.3. Engineering Application. The real engineering practice for the cofferdam described of the numerical experiment 2 in Section 3.2 is taken as an engineering application. As illustrated in Figure 6, the steel thin-walled cofferdam for bridge foundation construction was set up at $25.72^{\circ} \mathrm{N}$, $119.61^{\circ} \mathrm{E}$ in the China East Sea. The cofferdam was supported by 13 steel-concrete composite piles, of which the diameter is $2.2 \mathrm{~m}$. The piles were fully fixed on the seabed rocks. Wave pressure was measured using 11 CSW560 flush type pressure gauges, of which the measuring range is 0 to $200 \mathrm{kPa}$ and the measuring error is less than $0.5 \%$. In addition, for validation purposes, the wave elevations were also measured using a single-point SBY 2-1 ultrasonic wave gauge deployed $80 \mathrm{~m}$ away from the cofferdam, of which the measuring accuracy is $\pm 0.2 \mathrm{~m}$ for wave heights and $\pm 0.25 \mathrm{~s}$ for wave periods. The field measurement of an extreme wave event was conducted on the morning of September 29, 2015, when Typhoon Dujuan was passing through the studied sea area. During the measurement, the storm eye was around $119.40^{\circ} \mathrm{E}, 25.00^{\circ} \mathrm{N}$, which was about $80 \mathrm{~km}$ south from the tested cofferdam, as shown in Figure 7. The pressure at the storm center was $925 \mathrm{kPa}$ and the maximum wind speed of the storm was $35 \mathrm{~m} / \mathrm{s}$. Actual wave pressure data on the cofferdam were successfully collected during the extreme wave events. More information about configurations of the in-situ measurement can also be found in a previous study [27]. The measured pressure time series were used to estimate the directional wave spectrum using the presented approach.

\section{Result and Discussion}

\subsection{Numerical Experiment 1: Circular Cylinder}

4.1.1. Effect of Gauge Arrays. Previous studies show that the success of spectrum estimation heavily depends on the appropriate choice of gauge array and therefore, it is necessary to investigate the effect of the array on the estimations. Four different plans with a various number of gauges and array patterns are tested, as shown in Table 1. Estimation of directional spectra contains two aspects, i.e., estimation of spreading function $D(\theta)$ and estimation of spectral density $S(f) . S(f)$ representing the energy distribution is relatively easy to estimate while $D(\theta)$ containing directional 


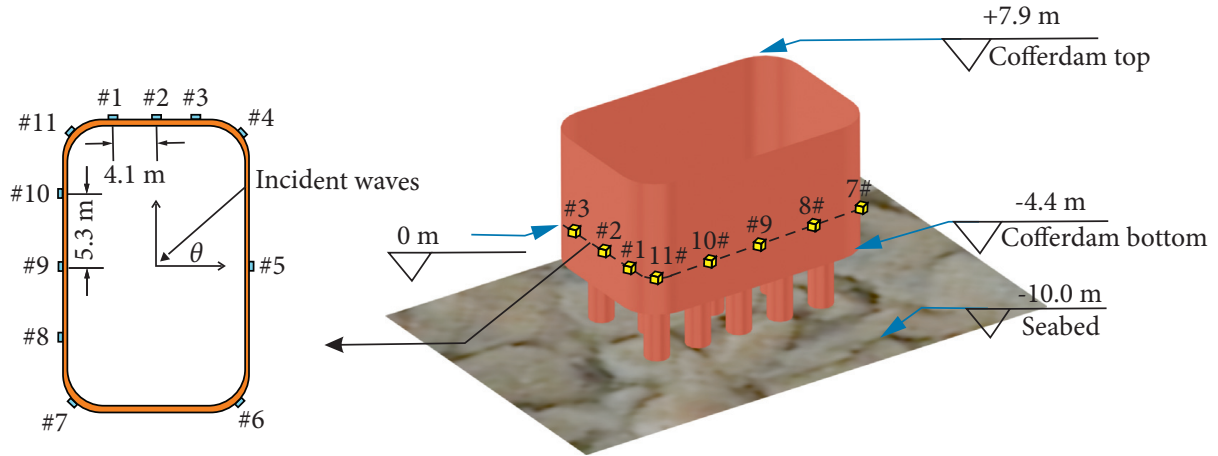

Figure 4: Cofferdam layout and deployment of pressure gauges.

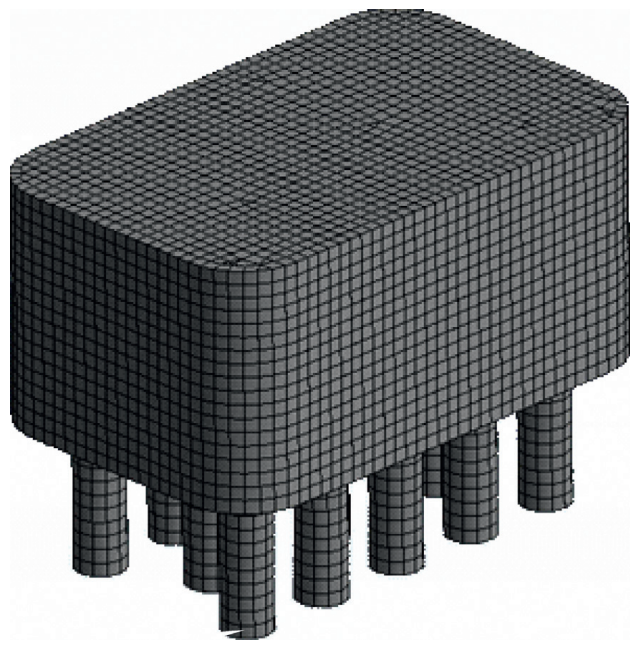

(a)

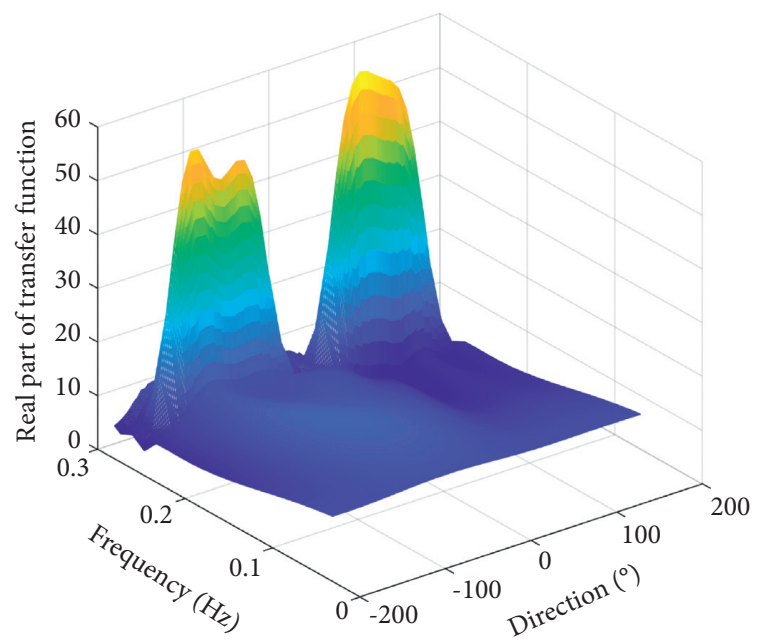

(c)

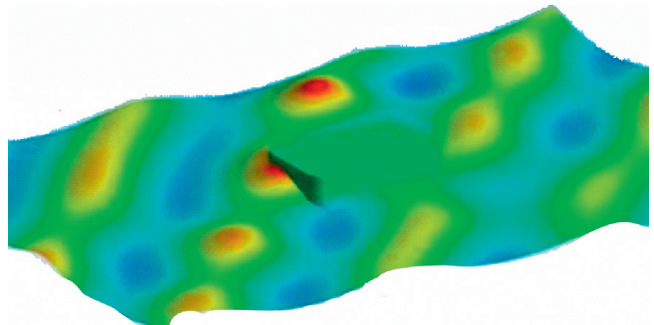

(b)

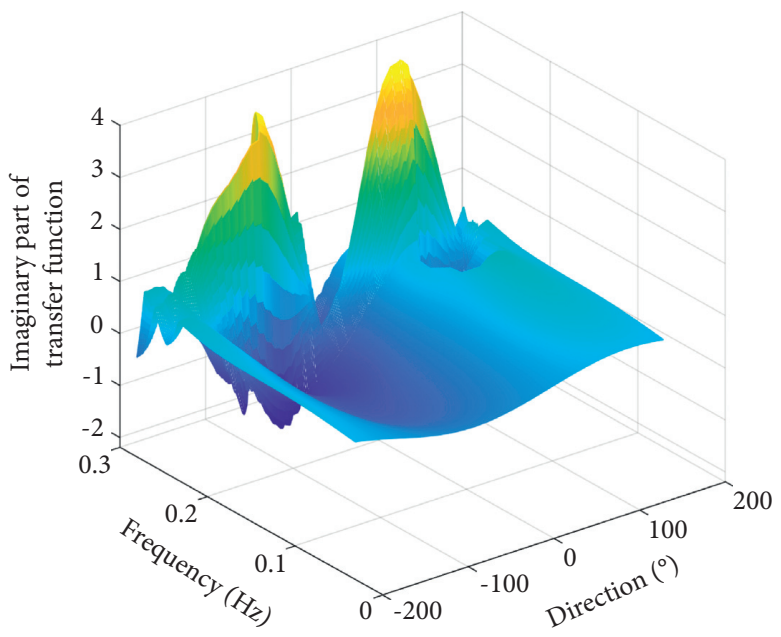

(d)

FiguRE 5: BEM model and pressure transfer function on the cofferdam: (a) panel mesh of cofferdam; (b) wave field simulation around the cofferdam; (c) real part of the pressure transfer function at \#4 pressure gauge; (d) imaginary part of the pressure transfer function at \#4 pressure gauge.

information is trickier and more important and hence draws more attention in previous studies $[4,6,28]$. Therefore, the estimation of $D(\theta)$ is a priority in this study and will be put more discussions. The corresponding estimated spreading functions $D(\theta)$ are illustrated in Figure 8. It can be observed that Plan 1, which employs 7 gauges, gives the most plausible result. Though Plans 2 and 3 reasonably predict the shape of the target spreading function, they slightly underestimate the 


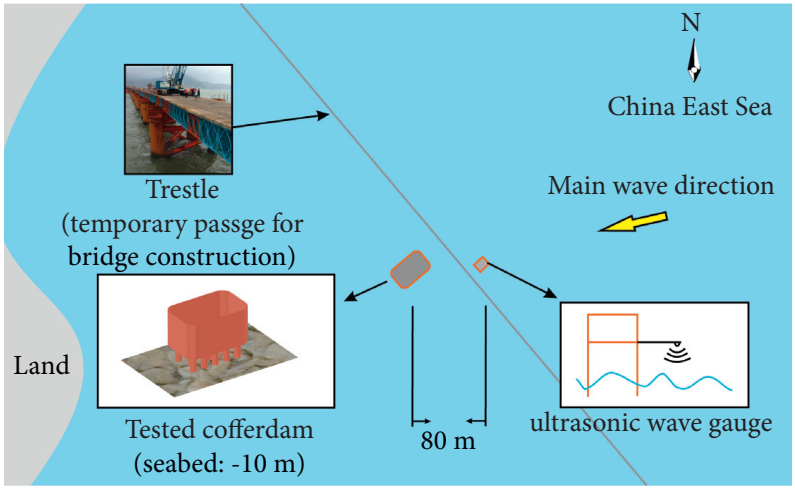

FIgURE 6: Layout of the measurement site.

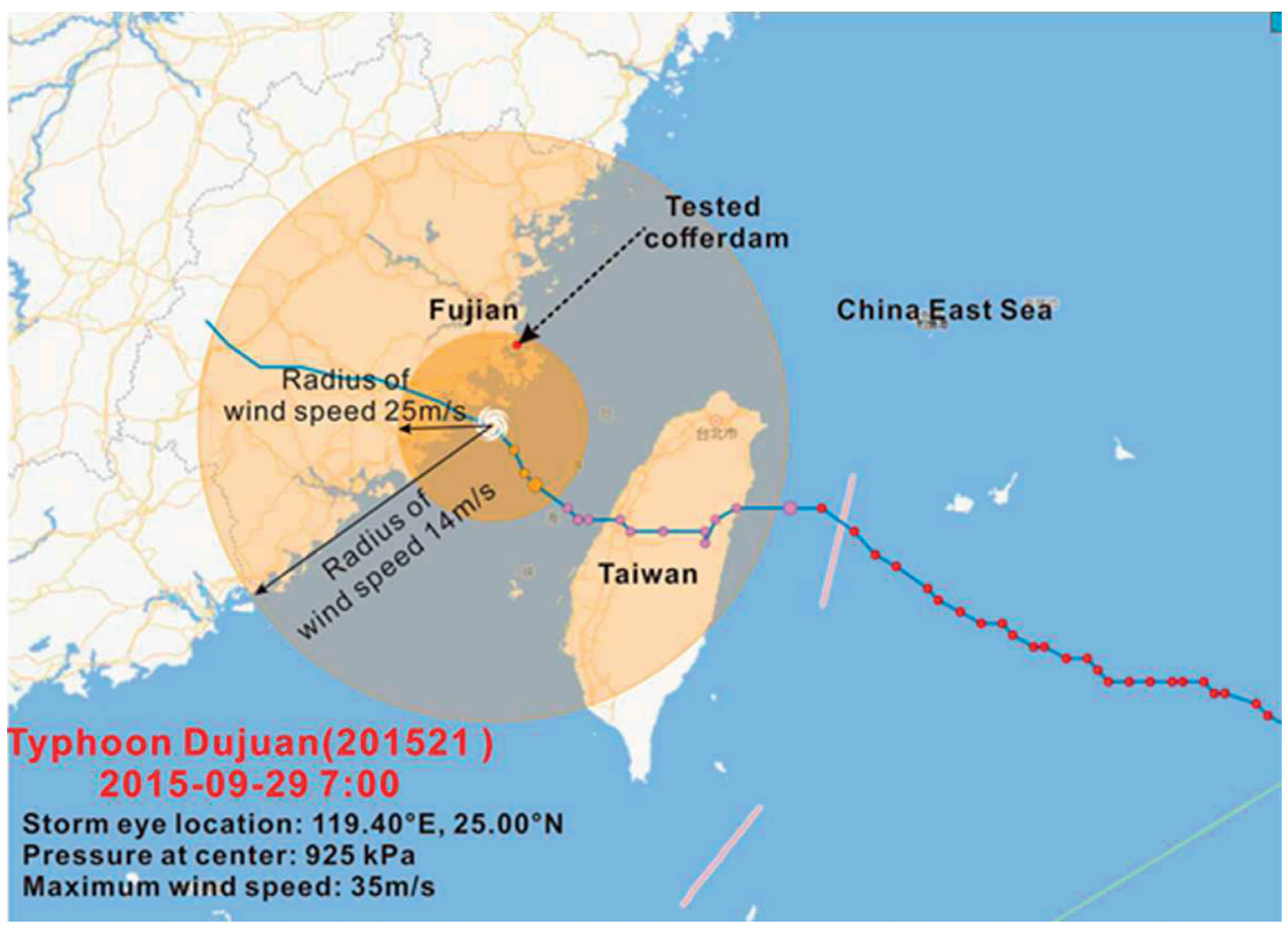

FIgURE 7: Location of the typhoon during the measurement.

TABLE 1: Different plans to test the effect of array pattern.

\begin{tabular}{lcc}
\hline Array pattern & Gauge no. & Number of employed gauges \\
\hline Array 1 & $4,3,2,1,12,11,10$ & 7 \\
Array 2 & $4,2,1,12,10$ & 5 \\
Array 3 & $3,1,11$ & 3 \\
Array 4 & $5,6,7,8,9$ & 5 \\
\hline
\end{tabular}

peaks. Plan 4 deviates significantly from the target and is not applicable. It is worthwhile to notice that in Plan 1, compared with the target directional function, the estimated spreading function has two small 'wings' in directions of $-180^{\circ}$ to $-90^{\circ}$ and $90^{\circ}$ to $180^{\circ}$. This phenomenon is due to the fact that the diffracted waves are a series of cylindrical waves which are scattered from the cylinder surface in all directions, as demonstrated in Figure 9(a) and Figure 1(b). The proposed approach separates all wave components and the side "wings" represent the diffracted wave components. Since we are only interested in the incident waves, the spreading function is cut off at the tails and only the main peak which represents the incident waves is retained, as explained in Figure 8(b). This cutting action is conducted in all the following results to manually eliminate the diffracted components.

To enhance the advantage of the proposed approach, the estimated results are compared with the conventional approach [5] (4), i.e., using pressure signals to estimate the directional spectrum without considering the diffraction effect, and the comparison is shown in Figure 10. It can be seen that the proposed approach successfully estimates the spreading function while the conventional approach 


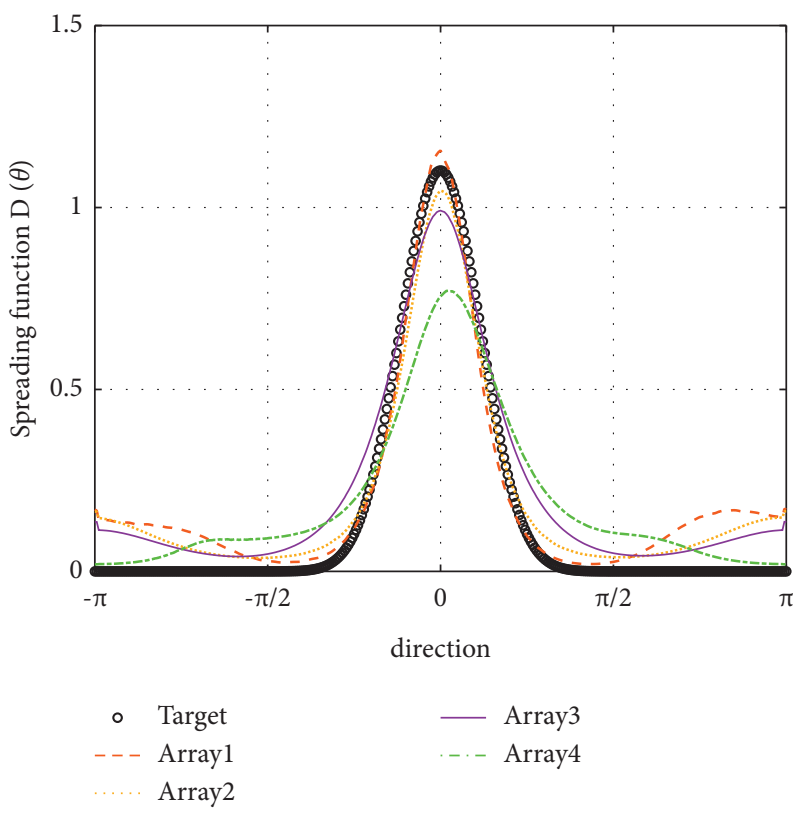

FIGURE 8: Estimated spreading function using different arrays.

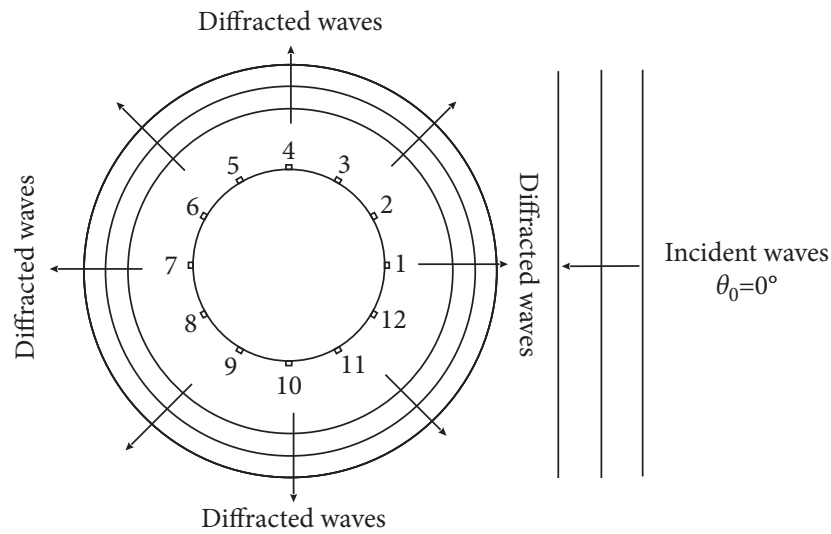

(a)

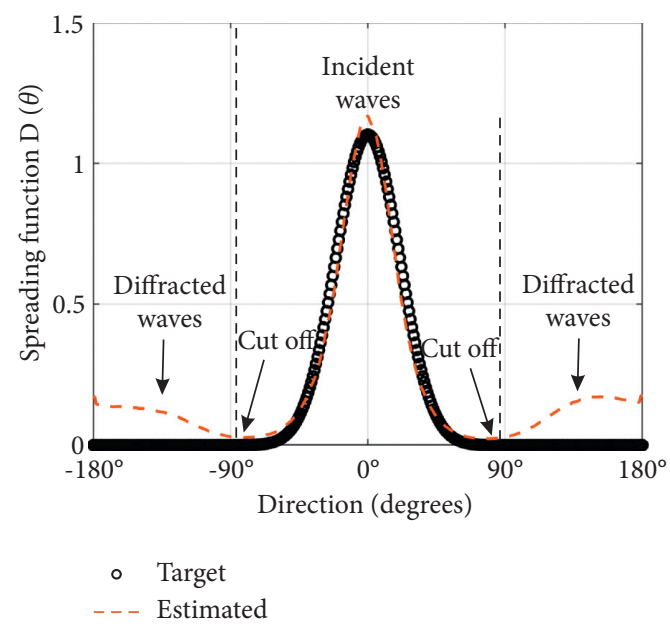

(b)

FIGURE 9: Separation of the incident and diffracted wave spectral components: (a) sketch of diffracted waves; (b) incident and diffracted spectral components.

underestimates the peak and overestimates the width of the spreading function because it cannot separate the coupled incident and diffracted wave components. With respect to the estimated spectral density $S(f)$, Figure 10(b) shows that the conventional approach significantly overestimates the spectral density while the presented approach aligns with the target well. Figure 11 illustrates the estimated three-dimensional directional spectra and shows that the conventional approach poses significant discrepancies of the direction and energy distribution in the estimated spectrum and overestimates the significant wave height and main wave direction. The presented approach agrees well with the target spectrum. It again proves that it is necessary to consider the diffraction effect in spectrum estimation and the conventional approach may lead to significant errors.
4.1.2. Effect of Spreading Coefficient. The spreading coefficient $s$ in the spreading function $D(\theta)$ dominates the width of wave direction distribution and represents different types of sea states. To test the ability of the proposed approach on spectrum estimation with different spreading coefficients $s$, stochastic wave pressure data with $s=10,25$, and 75 are generated and used to perform the spectrum estimation. According to Goda [29], these three typical spreading coefficients represent sea states of wind wave $(s=10)$, shortdistance decaying swell $(s=25)$, and long-distance decaying swell $(s=75)$. The estimated spectra are compared with the target and presented in Figure 12. Note that all estimations are performed using Array 1 validated in the previous section, and diffracted components are eliminated. All spectra are normalized by the maximal value in the target 


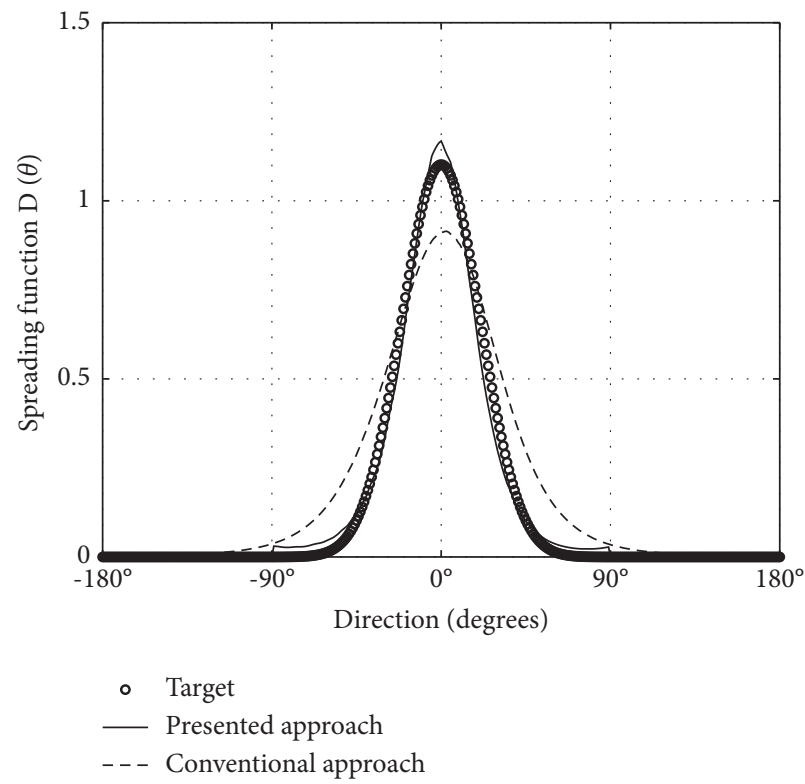

(a)

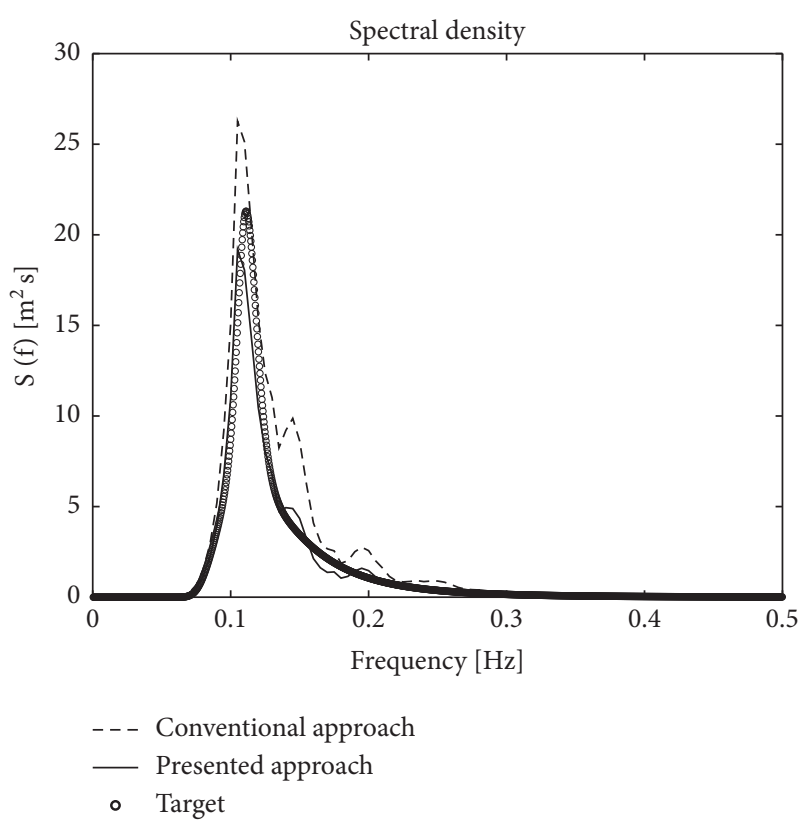

(b)

FIGURE 10: Comparison of estimated spreading function and spectral density: (a) spreading function; (b) spectral density.

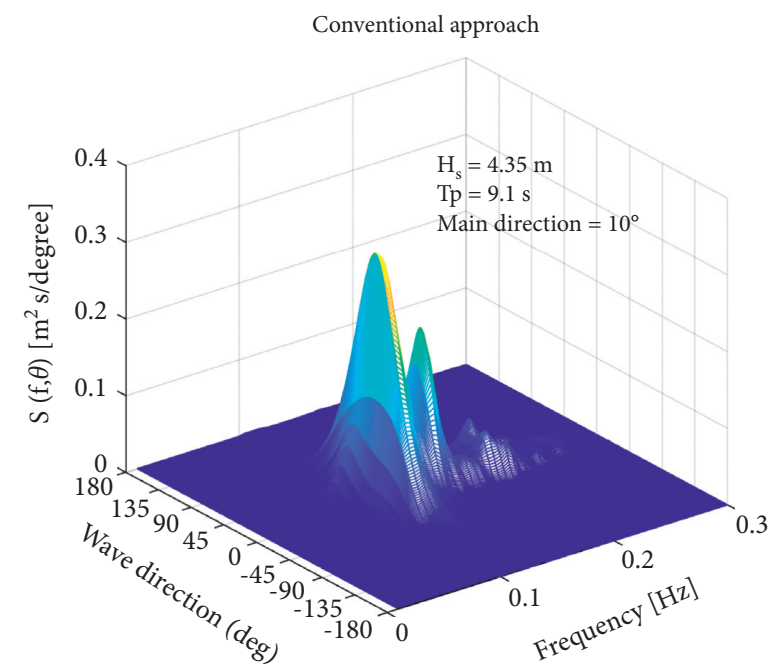

(a)

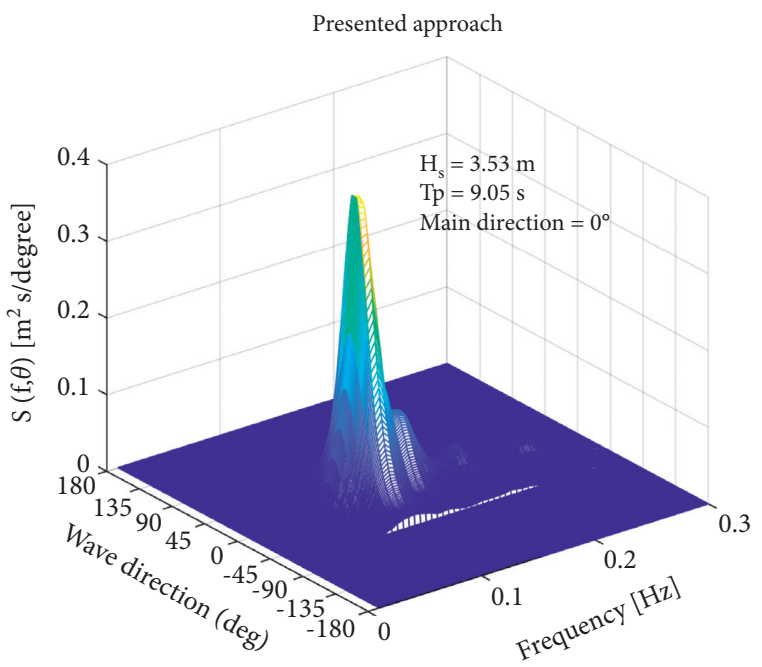

(b)

Figure 11: Continued. 


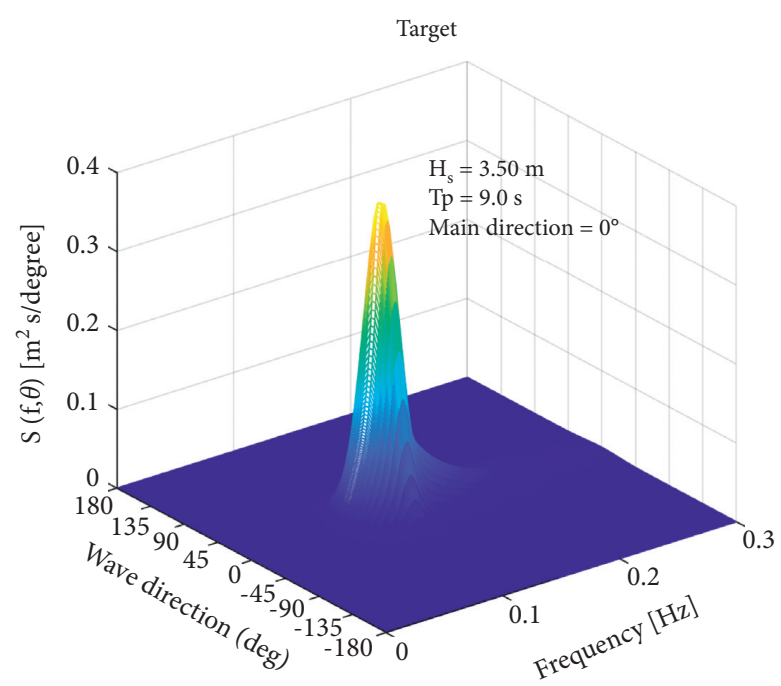

(c)

FIGURE 11: Comparison of estimated directional wave spectra: (a) conventional approach; (b) presented approach; (c) target spectrum.

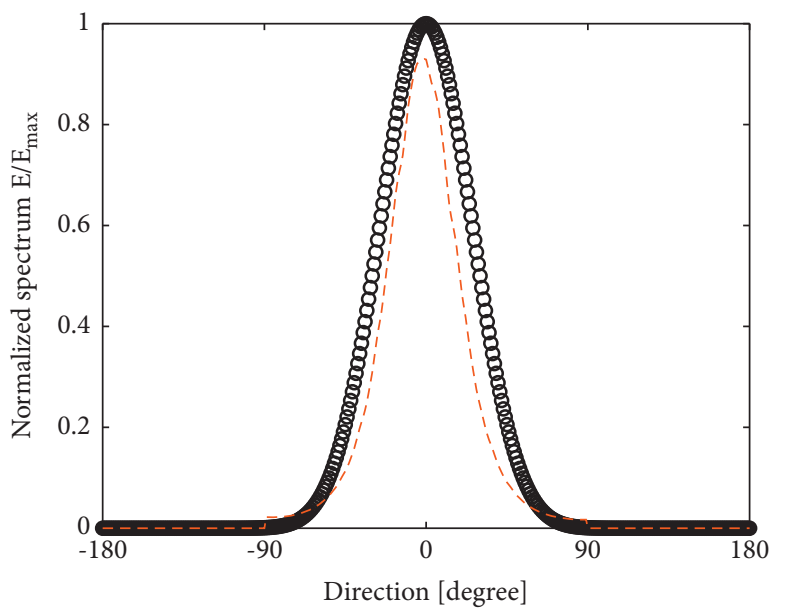

○ Target $(s=10)$

- - Estimated

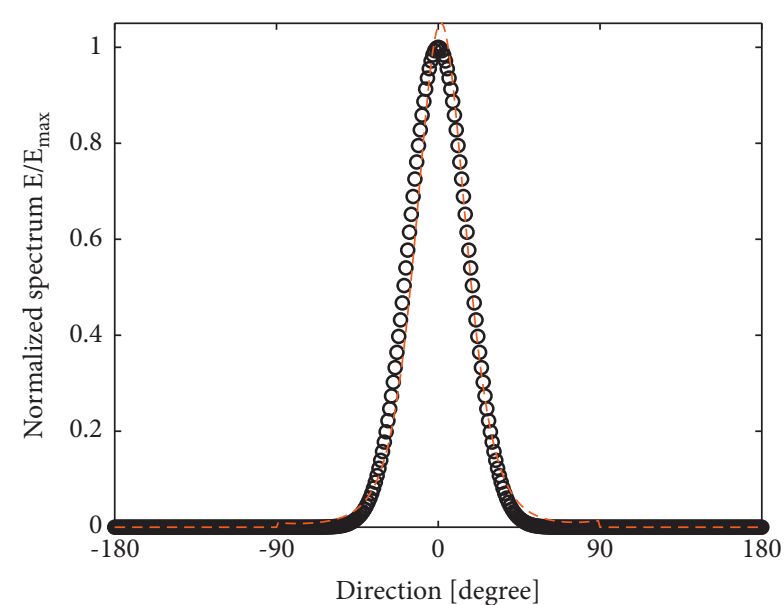

○ Target $(\mathrm{s}=25)$

- - - Estimated

(a)

(b)

Figure 12: Continued. 


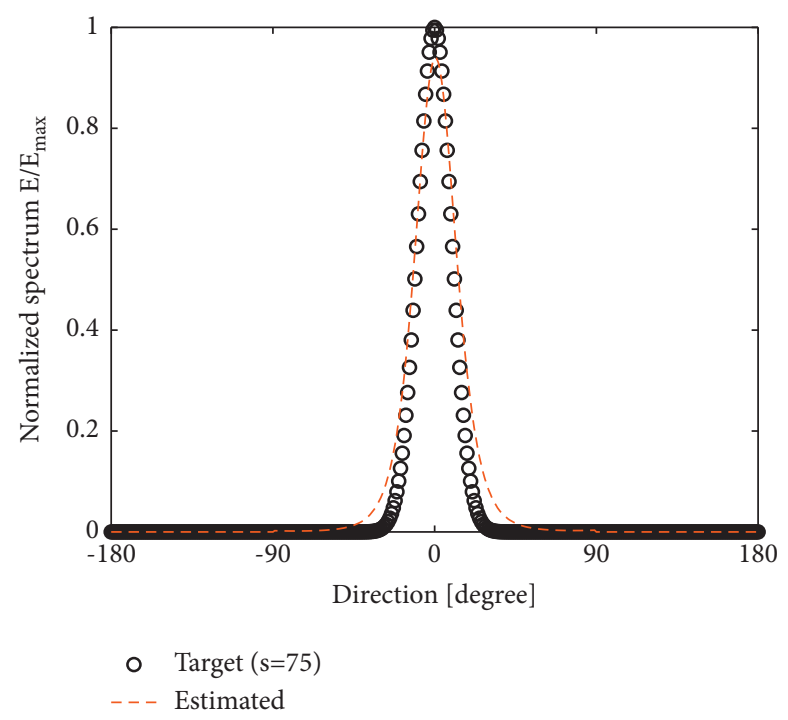

(c)

FIGURE 12: Estimated spreading function under various sea states: (a) spreading coefficient $(\mathrm{s})=10$; (b) spreading coefficient (s) = 25; (c) spreading coefficient $(s)=75$.

spectra, i.e., $E(\theta, f) / E(\theta, f)_{\max }$ in order to have a better view for comparison. It shows that the proposed approach estimates reasonable directional spectra for all spreading coefficients, though it seems to slightly underestimate the width of directional spreading for scenario $s=10$, and some slight deviations in the peak estimations. Nevertheless, it shows that the presented approach can give plausible directional estimations under various sea states.

4.1.3. Effect of Background Noise. In engineering practice, measurement data inevitably contain background noise, and therefore is necessary to investigate the effect of noise on estimating performance. Noise level is usually measured by signal-noise-ratio (SNR), which is defined as the ratio of signal power to noise power:

$$
\mathrm{SNR}=\frac{P_{\text {Signal }}}{P_{\text {Noise }}}
$$

The unit of SNR is $\mathrm{dB}$ and a large value of SNR indicates good quality signals. In this study, artificial Gaussian white noise is mixed into the simulated pressure signals to generate pressure data with noise, and pressure data with various levels of noise ( $\mathrm{SNR}=100,50,20$, and 10) are fed to the estimation approach to test its performance against noise. The estimated directional spectra are normalized and shown in Figure 13. Note that to illustrate the original estimations, the diffraction components, i.e., the side wings, are not cut off in the results. It indicates that the estimated results have a reasonable agreement with the target when $\mathrm{SNR}=100,50$, and 20 , while it underestimates the peak value and overestimates the width of spreading function when $S N R=10$. With the increasing background noise, the accuracy of estimated spectra drops and leads to deviations in directional spreading. However, in general, the results reveal that the

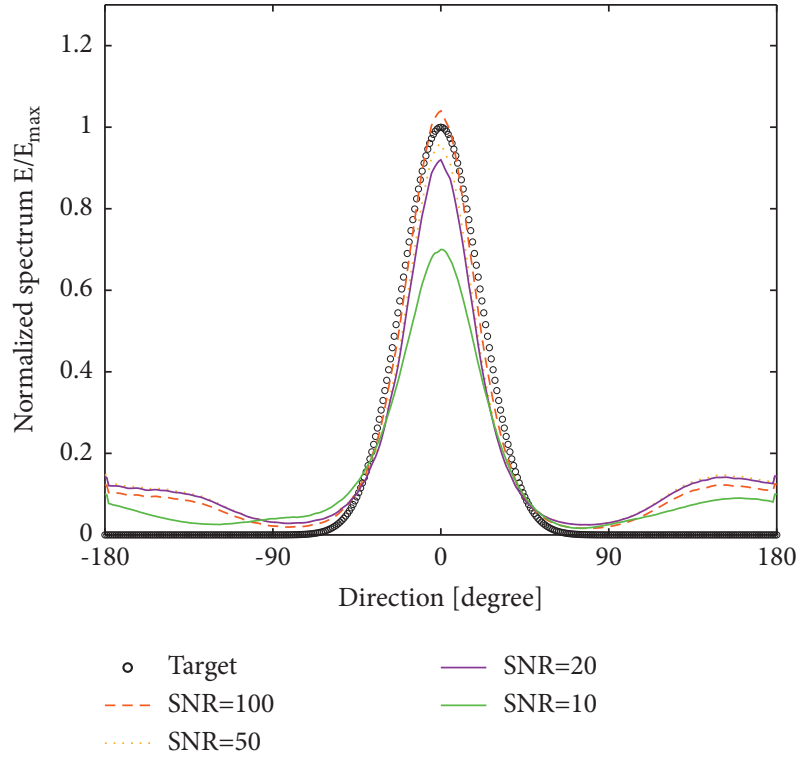

FIGURE 13: Estimated spectra under various levels of background noise in pressure data with $S N R=100,50,20$, and 10 .

presented method performs well against background noise which should attribute to the good noise resistance of the BDM itself. (11) shows that BDM has considered a Gaussian error term in its governing formulations and hence can, to a considerable degree, eliminate the background noise and gives favorable estimation. The good performance in noise resistance of the method provides a basis for further application into field measurement.

4.2. Numerical Experiment 2: Quasirectangle Cofferdam. This numerical experiment is used as a proof-of-concept study and aims to further deploy into a real in-situ 

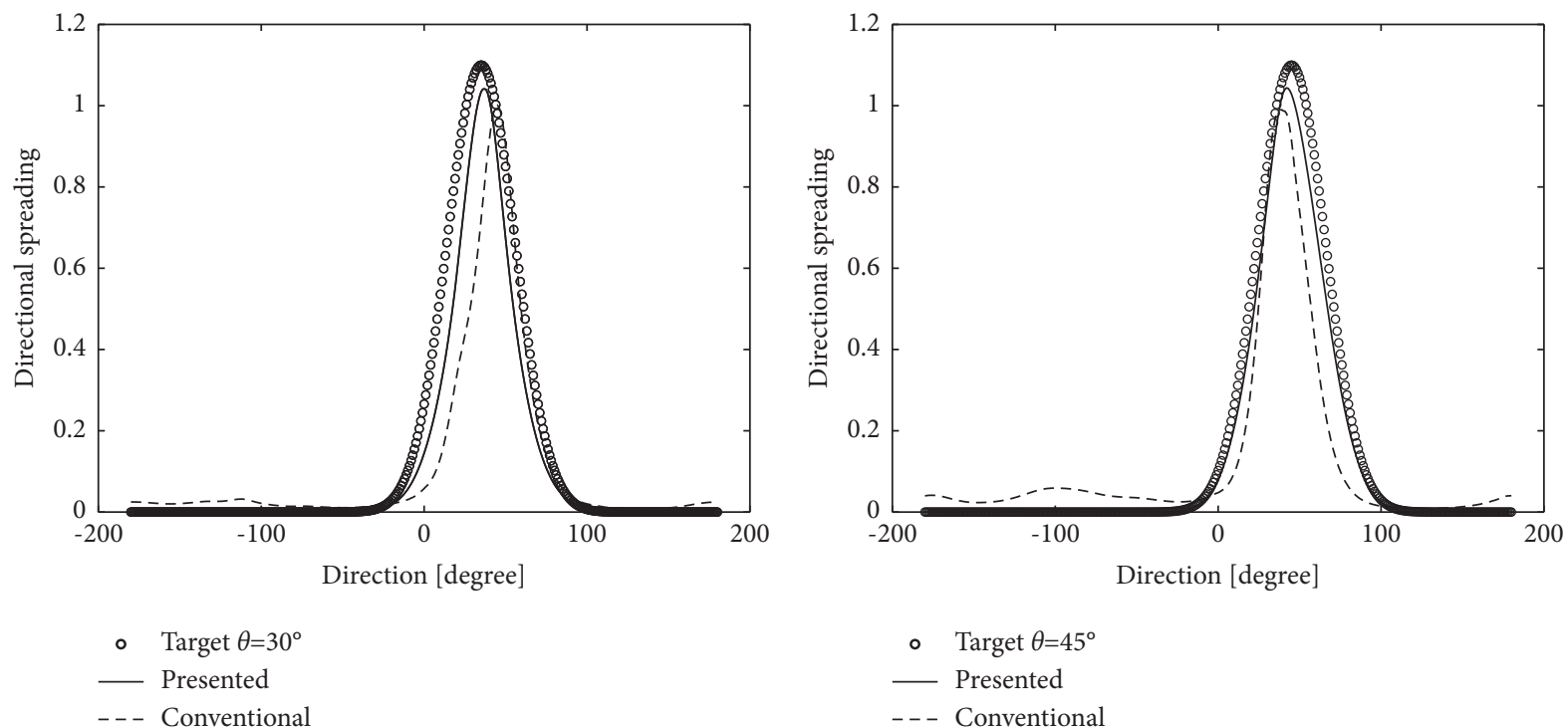

(a)

(b)

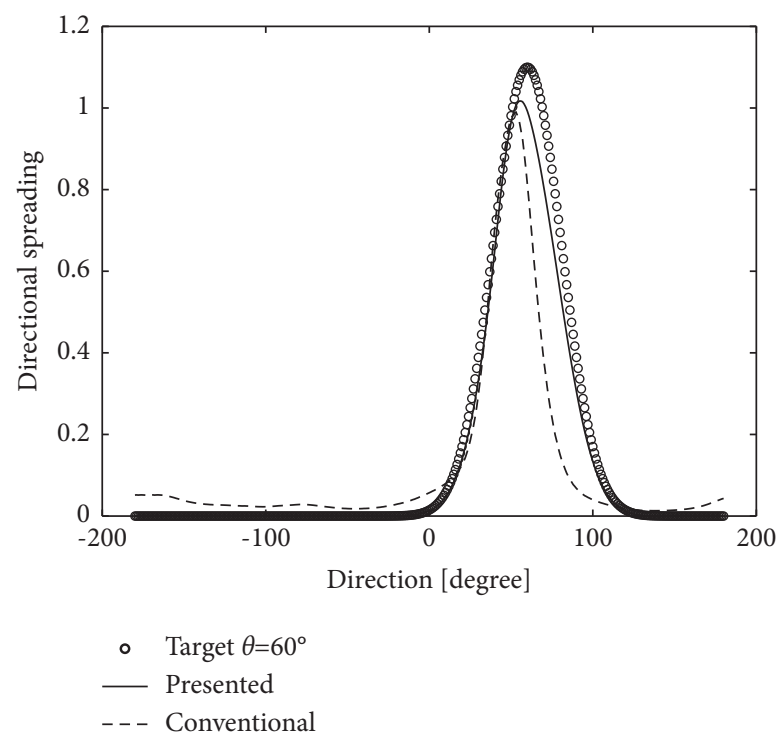

(c)

FIGURE 14: Estimated spreading functions under various wave directions: (a) $\theta=30^{\circ}$; (b) $\theta=45^{\circ}$; (c) $\theta=60^{\circ}$.

application. By performing the same procedure in Section 4.1 , the effect of gauge array, spreading coefficients and background noise can be tested to choose the best array and validate the presented approach. For the sake of conciseness, the similar results are not illustrated and discussed again. This section will pay more attentions to the effect of wave direction on spectrum estimation. Since the cofferdam is a noncentrosymmetric geometry and the effect of incident wave directions on spectrum estimation should be carefully discussed. In this case, 5 gauges including \#1, \#2, \#4, \#5, and \#6 are taken as the estimation array. The input simulated pressure signals are mixed with Gaussian white noise with $\mathrm{SNR}=20$. The estimated spreading function $D(\theta)$ and spectral density $S(f)$ regarding various incident wave directions of $30^{\circ}, 45^{\circ}$, and $60^{\circ}$ are illustrated in Figures 14 to 17, respectively. Note that according to the long-term in-site observation, the main wave directions at the studied cofferdam concentrate in a relative narrow range about $30^{\circ}-60^{\circ}$ due to the local seabed topography and opening direction of the strait, as shown in Figures 6 and 7. Therefore, only wave directions of $30^{\circ}, 45^{\circ}$, and $60^{\circ}$ are tested and discussed. The estimated results using the presented approach are also compared with the conventional approach to enhance the effect of diffracted waves. Figure 14 shows that, in general, the presented approach reasonably estimates most of the spreading functions and main wave directions, while some slight discrepancies in main directions are still observed in case $\theta=30^{\circ}$ and $\theta=60^{\circ}$ and the presented approach underestimates the width of directional functions in case $\theta$ $=30^{\circ}$. Nevertheless, the presented approach still shows advantages over the conventional approach, which obviously underestimates the width of the spreading function and 

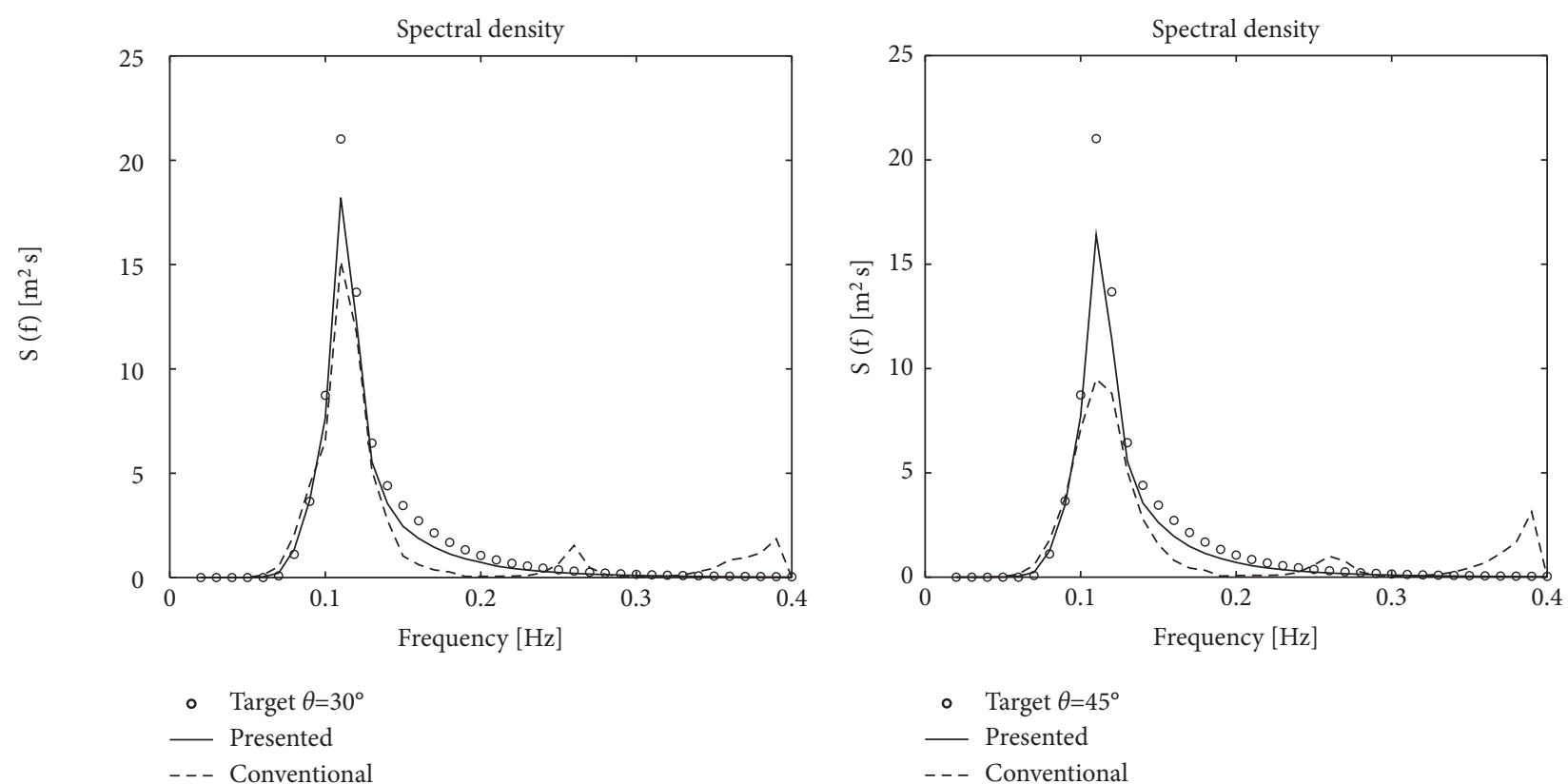

- Target $\theta=45^{\circ}$

— Presented

- - - Conventional

(a)

(b)

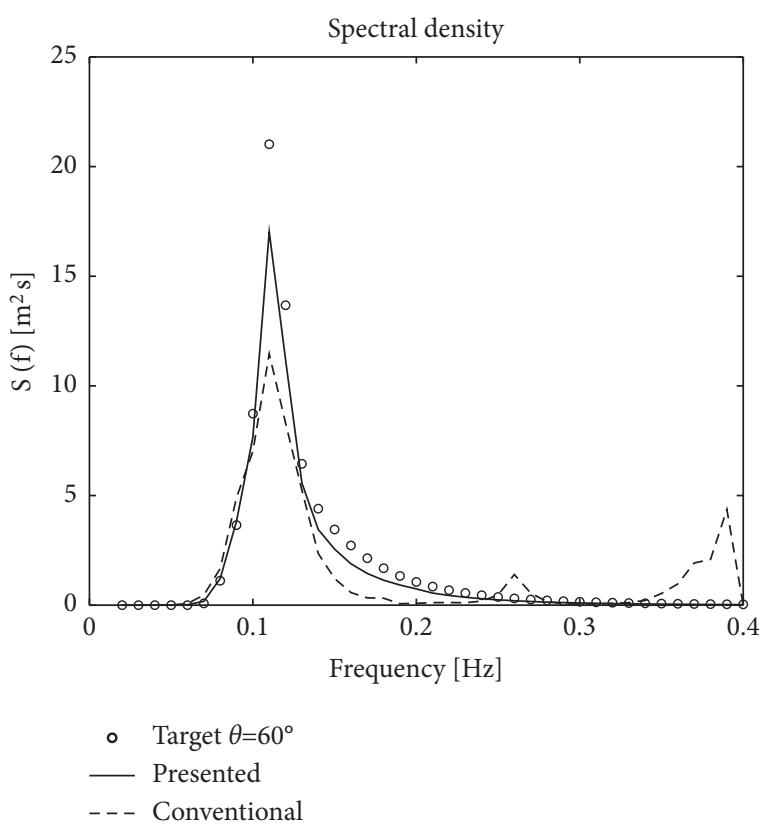

(c)

Figure 15: Estimated spectral density under various wave directions: (a) $\theta=30^{\circ}$; (b) $\theta=45^{\circ}$; (c) $\theta=60^{\circ}$.

predicts inaccurate main directions due to a lack of ability to separate incident and diffracted wave components. In Figure 15, it reveals that the conventional approach significantly underestimates the peak energy and spectral width. The conventional approach also causes some unnecessary high frequency components, which could impose errors in wave spectra for real engineering applications. Compared with conventional approach, the presented approach obviously improves the spectral density estimation by considering the coupled relationship between incident and diffracted waves. It indicates that the presented approach can yield appreciable spectrum estimations for various wave directions and provides basis for further application into in-situ measurement.

4.3. Application: Field Measurement Data Analysis. A series of wave pressure measurement data during typhoon Dujuan in 2015 on an actual quasirectangle cofferdam, which has been discussed in Section 4.3, is applied to estimate the directional wave spectrum. Figure 16 shows four samples of estimated three-dimensional directional spectra collected at 


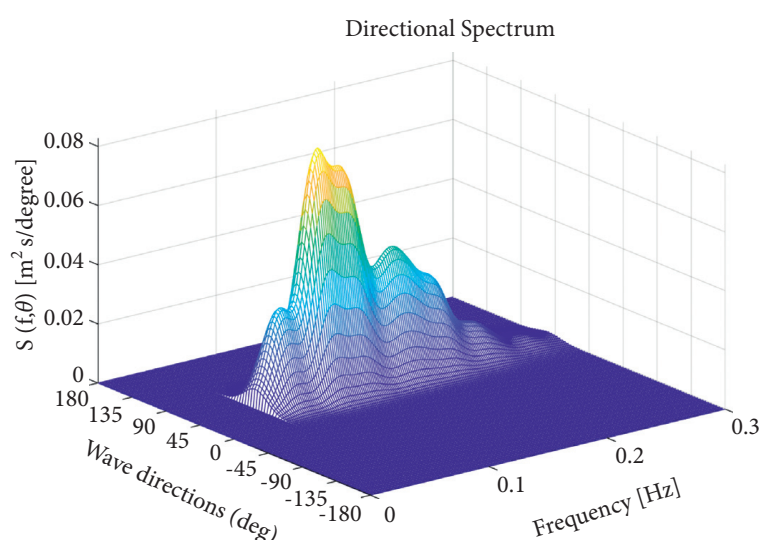

(a)

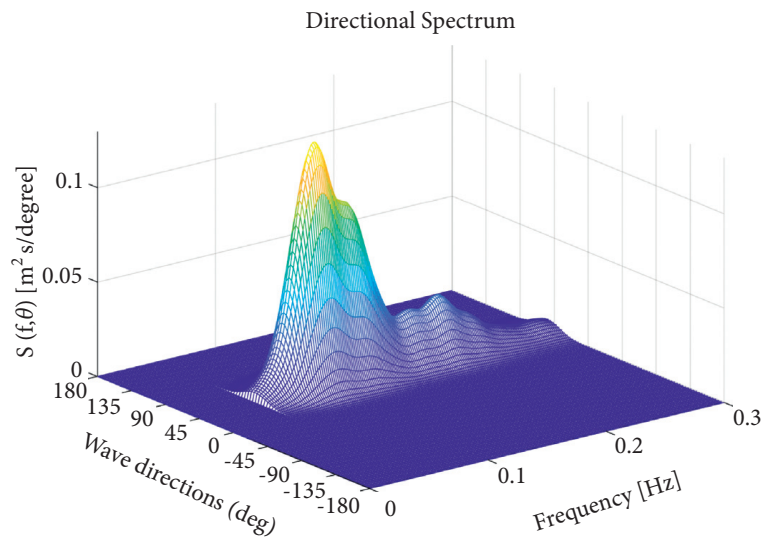

(c)

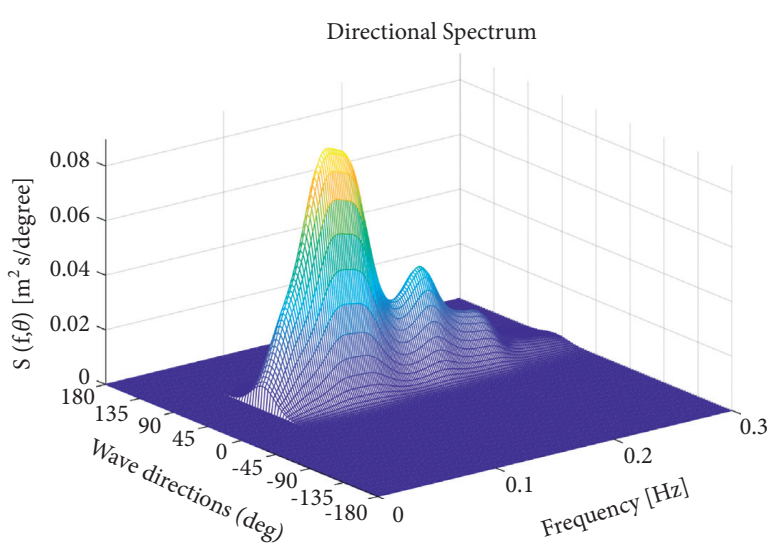

(b)

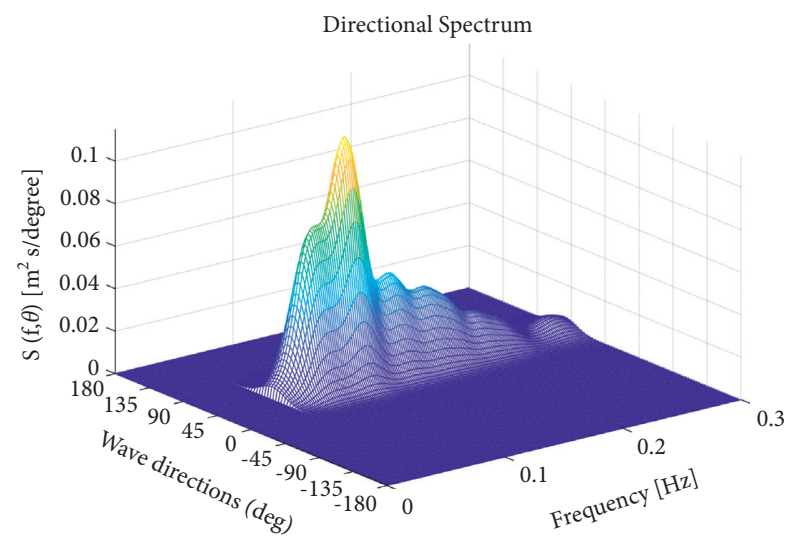

(d)

FIGURE 16: Estimated directional spectra at different moments during Typhoon Dujuan: (a) 2015/09/29 0:00; (b) 2015/09/29 0:10; (c) 2015/ 09/29 1:10; (d) 2015/09/29 2:00.

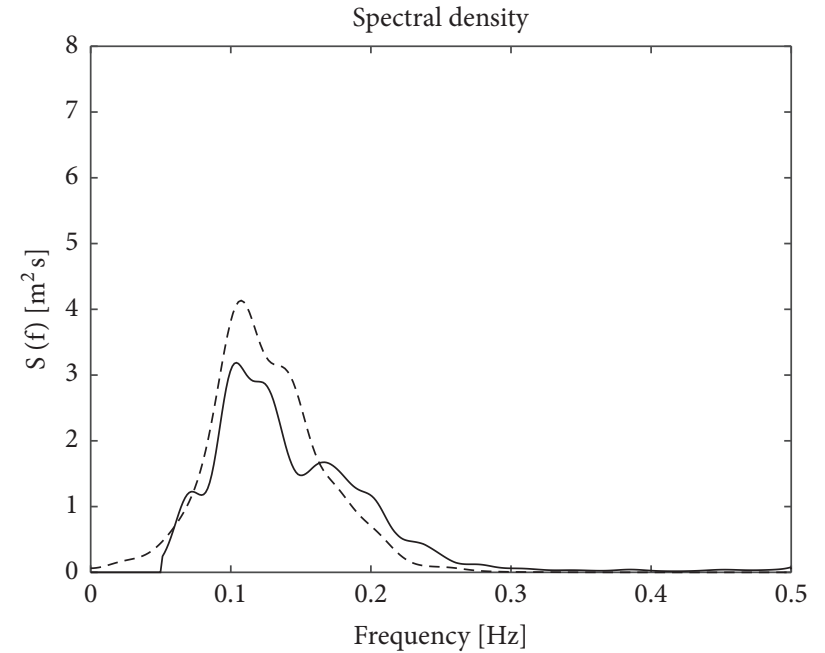

- Estimated

- - - Measured

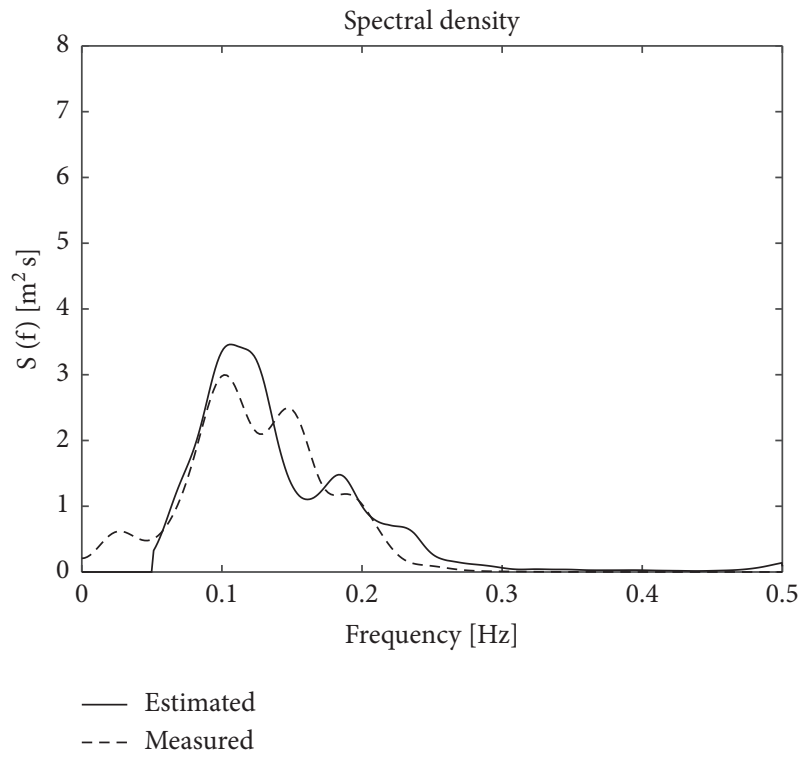

(b)

FIgUre 17: Continued. 


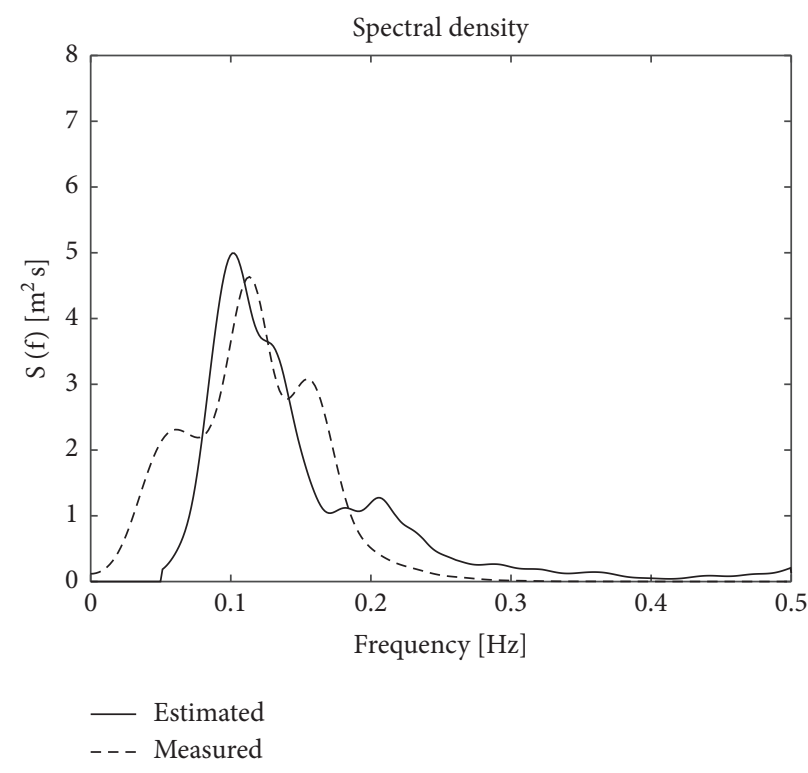

(c)

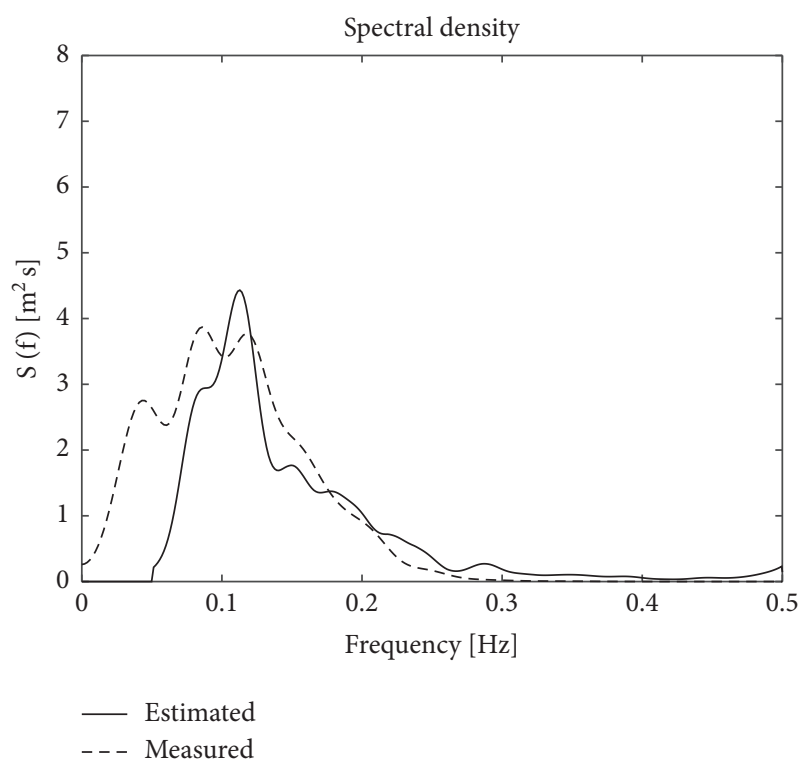

(d)

FiguRE 17: Estimated and measured spectral density at different moments during Typhoon Dujuan: (a) 2015/09/29 0:00; (b) 2015/09/29 0: 10; (c) $2015 / 09 / 291: 10$; (d) 2015/09/29 2:00.

different moments during 2015/09/29 0:00 to 2015/09/29 02: 00. It indicates that the presented approach works robustly on the measurement samples. The measured directional spectra reveal that during this typhoon event, the main wave directions are $\theta=49^{\circ}-61^{\circ}$ and the fitting spreading coefficient $s=22.5-28.8$, representing sea states transiting from wind waves to short distance decaying swells. To validate the results, the estimated spectral density is compared with measured spectral density, which is collected using a single-point style ultrasonic wave gauge, as introduced in Section 3.3 and Figure 6. Note that the wave gauge is only capable of analyzing two-dimensional spectra because the elevation data at a single point is insufficient for wave directional estimation. Nevertheless, the comparison of spectral density can, to a certain extent, prove the consistency and validity of the presented approach in spectrum estimation. Figure 17 demonstrates the comparison of estimated and measured spectral density. It indicates that the presented approach estimates the spectral density with a reasonable agreement, though some deviations are found in low frequency range. It should be noted that the wave gauge is about $80 \mathrm{~m}$ away from the cofferdam, and the spatial variability of wave spectral characteristics may cause these deviations in estimated and measured spectra, which is a reasonable explanation of deviations in the comparison of spectral density in Figure 17. In general, the presented approach works well in in-situ measurement and can estimate the wave spectra with plausible accuracy. It provides proof-of-concept evidence for potential applications in more offshore engineering practice.

\section{Conclusion}

In the present study, the diffraction wave theory is introduced into the estimation of directional wave spectrum to consider the effect of diffracted waves using array pressure data from existing pressure gauges on structures. Two numerical experiments are conducted as proof-of-concept studies to test and evaluate the feasibility of the presented approach under various structures and wave scenarios. The performance of the presented approach using various gauge arrays, different wave directions and spreading coefficients, and multiple levels of background noise is evaluated and discussed, respectively. The presented approach is also deployed into an in-situ measurement application and compared with wave observation data to test its validity in engineering practice.

The numerical experiments show the validity of the presented approach. By using the correct transfer function, either analytical or numerically modeled, the presented approach can estimate the directional spectra under various wave scenarios with a plausible agreement. The gauge array significantly affects the spectrum estimation and a sensitive test is necessary. A high level of background noise can lead to deviations in estimated spectra while the presented approach has a good noise resistance which should attribute to the BDM method. The presented approach shows apparent advantages over the conventional approach in diffracted and incident wave field, which indicates that considering the diffraction effect in spectrum estimation is essential. The presented approach works well in in-situ applications and can estimate the wave spectra with plausible accuracy. The estimated spectra during Typhoon Dujuan in 2015 show that the sea states which the cofferdam was actually encountering have the main wave directions of $49^{\circ}-61^{\circ}$ and the fitting spreading coefficient $s=22.5-28.8$, representing sea states transiting from wind waves to short distance decaying swells. The experiment results and engineering application provide proof-of-concept evidence of the presented approach for potential applications in more engineering practice. 
The presented approach uses existing pressure gauges which are a part of the structural health monitoring (SHM) system, is a low-cost alternative for spectrum estimation, and shows potentials and advantages for engineering practice. However, this article aims at a proof-of-concept study and some limitations should be pointed out. At present, only a uni-modal incident wave situation is considered. If the incident waves are bimodal, i.e., the superposition of two or more sea states, the diffracted components may fall into the incident directional range, and therefore, the spectral components of the incident and diffracted waves are mixed and cannot be manually eliminated. This case requires autorecognition and separation of diffracted and incident components, which may call for a more advanced mathematical model in spectrum estimation. Another inherent difficulty in separating incident and diffracted waves is that diffracted waves are usually cylindrical waves which means they may distribute in all directions. When the incident waves spread in a wide range, they inevitably overlap over the diffracted waves and may affect the estimated spectrum. Selection for the most suitable gauge array under these scenarios should be very careful and requires further investigation and knowledge. Note that the diffraction effect is only significant for large-scale structures and becomes negligible for components like offshore structure legs. In the future, more experimental or measured data should be collected to further test the validity of the presented method under more complex sea states. Some advanced methods [30] are worthwhile to implement to further improve the accuracy and smoothness in estimated spectra.

\section{Data Availability}

The spectral data presented in the figures are available from the corresponding author upon request.

\section{Conflicts of Interest}

The authors declared no potential conflicts of interest with respect to the research, authorship, and/or publication of this article.

\section{Acknowledgments}

This work was supported financially by the National Natural Science Foundation of China (Grants no. 52008349), Postdoctoral Science Foundation of China (Grants nos. 2020M683356, 2021T140573), and the Fundamental Research Funds for the Central Universities (Grants no. 2682021CX004).

\section{References}

[1] J. Liu, A. Guo, H. Li, and H. Hu, "Methodology for wave force monitoring of bottom-mounted cylinder using the measurement of the wave surface elevation around the body surface," Journal of Fluids and Structures, vol. 78, pp. 197-214, 2018.

[2] Z. Ti, M. Zhang, Y. Li, and K. Wei, "Numerical study on the stochastic response of a long-span sea-crossing bridge subjected to extreme nonlinear wave loads," Engineering Structures, vol. 196, Article ID 109287, 2019.

[3] R. MacCamy and R. A. Fuchs, "Wave Forces on Piles: A Diffraction Theory," in Corps of Engineers, Beach Erosion Board, Washington, DC, USA, 1954.

[4] N. Hashimoto, K. Kobune, N. Hashimoto, and K. Kobune, "Directional Spectrum Estimation from a Bayesian Approach," in Proceedings of the 21th ICCE, pp. 62-76, ASCE, Malaga, Spain, 1988.

[5] G. L. Howell, "Shallow water directional wave gages using short baseline pressure arrays," Coastal Engineering, vol. 35, no. 1-2, pp. 85-102, 1998.

[6] M. Isobe and K. Kondo, "Method for estimating directional wave spectrum in incident and reflected wave field," in Proceedings of the 19th ICCE, pp. 467-483, Houston, TX, USA, 1984.

[7] Y. U. Yu, L. M. Shao, and Dalian, "Estimation of directional spectrum and reflection coefficient of incidentand reflected waves in the phase-locked wave field," Ocean Engineering, vol. 21, pp. 34-41, 2003.

[8] C. Lee, J.-S. Jung, and M. C. Haller, "Asymmetry in directional spreading function of random waves due to refraction," Journal of Waterway, Port, Coastal, and Ocean Engineering, vol. 136, no. 1, pp. 1-9, 2010.

[9] J. Liu, Q. Fang, A. Guo, and H. Li, "Real-time nonlinear cylinder wave force reconstruction in stochastic wave field considering second-order wave effects," Journal of Fluids and Structures, vol. 98, Article ID 103132, 2020.

[10] J. M. Nichols, "Structural health monitoring of offshore structures using ambient excitation," Applied Ocean Research, vol. 25, no. 3, pp. 101-114, 2003.

[11] S. F. Barstow, J.-R. Bidlot, S. Caires, M. A. Donelan, W. M. Drennan, and H. Dupuis, "Measuring and Analysing the Directional Spectrum of Ocean Waves," COST Office, 2005.

[12] H. E. Krogstad and S. F. Barstow, "Recent Advances in Wave Measurement Technology," in Proceedings of the 9th International Offshore and Polar Engineering Conference, Brest, France, 1999.

[13] N. F. Barber, "Finding the direction of travel of sea waves," Nature, vol. 174, no. 4440, pp. 1048-1050, 1954.

[14] B. Strong, B. Brumley, E. Terray, and G. W. Stone, "The performance of ADCP-derived directional wave spectra and comparison with other independent measurements," in Proceedings of the OCEANS 2000 MTS/IEEE Conference and Exhibition Conference Proceedings (Cat No 00CH37158), pp. 1195-1203, IEEE, Providence, RI, USA, september 2000.

[15] M. Isobe, K. Kondo, and K. Horikawa, "Extension of MLM for estimating directional wave spectrum," Proc Symp on Description and modeling of directional seas, pp. 1-15, 1984.

[16] A. Lygre and H. E. Krogstad, "Maximum entropy estimation of the directional distribution in ocean wave spectra," Journal of Physical Oceanography, vol. 16, no. 12, pp. 2052-2060, 1986.

[17] U. D. Nielsen, "Estimations of on-site directional wave spectra from measured ship responses," Marine Structures, vol. 19, no. 1, pp. 33-69, 2006.

[18] E. A. Tannuri, P. C. Mello, J. S. Sales, A. N. Simos, and V. L. F. Matos, "Estimation of directional wave spectrum using a wave-probe array," Marine Systems \& Ocean Technology, vol. 3, no. 2, pp. 123-129, 2007.

[19] Y. Goda, "Random seas and design of maritime structures," Advanced Series on Ocean Engineering, World Scientific, vol. 33, World Scientific, Singapore, 3 edition, 2010. 
[20] S. Draycott, T. Davey, D. M. Ingram, A. Day, and L. Johanning, "The SPAIR method: isolating incident and reflected directional wave spectra in multidirectional wave basins," Coastal Engineering, vol. 114, pp. 265-283, 2016.

[21] T. Iseki and K. Ohtsu, "Bayesian estimation of directional wave spectra based on ship motions," Control Engineering Practice, vol. 8, no. 2, pp. 215-219, 2000.

[22] T. Iseki and D. Terada, "Bayesian estimation of directional wave spectra for ship guidance system," International Journal of Offshore and Polar Engineering, vol. 12, 2002.

[23] E. A. Tannuri, J. V. Sparano, A. N. Simos, and J. J. Da Cruz, "Estimating directional wave spectrum based on stationary ship motion measurements," Applied Ocean Research, vol. 25, no. 5, pp. 243-261, 2003.

[24] Z. Ti, Y. Li, and S. Qin, "Numerical approach of interaction between wave and flexible bridge pier with arbitrary cross section based on boundary element method," Journal of Bridge Engineering, vol. 25, 2020.

[25] M. Donelan, A. Babanin, E. Sanina, and D. Chalikov, "A comparison of methods for estimating directional spectra of surface waves," Journal of Geophysical Research: Oceans, vol. 120, no. 7, pp. 5040-5053, 2015.

[26] N. Hashimoto, T. Nagai, and T. Asai, "Extension of the maximum entropy principle method for directional wave spectrum estimation," European Journal of Pain, vol. 11, pp. 466-467, 1994.

[27] Z. Ti, K. Wei, S. Qin, D. Mei, and Y. Li, "Assessment of random wave pressure on the construction cofferdam for seacrossing bridges under tropical cyclone," Ocean Engineering, vol. 160, pp. 335-345, 2018.

[28] N. Hashimoto, T. Nagai, and T. Asai, "Extension of the maximum entropy principle method for directional wave spectrum estimation," in Proceedings of the Symposium on Description and Modeling of Directional seas, Paper No. A-6, Technical University of Denmark, Kongens Lyngby, Denmark, pp. 1-15, 1984.

[29] Y. Goda, "Statistical variability of sea state parameters as a function of wave spectrum," Coastal Engineering in Japan, vol. 31, no. 1, pp. 39-52, 1988.

[30] U. D. Nielsen, "Introducing two hyperparameters in Bayesian estimation of wave spectra," Probabilistic Engineering Mechanics, vol. 23, no. 1, pp. 84-94, 2008. 